\title{
Single molecule translocation in smectics illustrates the challenge for time-mapping in simulations on multiple scales
}

\author{
Biswaroop Mukherjee, ${ }^{1, a)}$ Christine Peter, ${ }^{2, a)}$ and Kurt Kremer ${ }^{3, a)}$ \\ ${ }^{1}$ S. N. Bose National Centre for Basic Sciences, Kolkata 700098, India \\ ${ }^{2}$ Department of Chemistry, University of Konstanz, 78547 Konstanz, Germany \\ ${ }^{3}$ Max-Planck-Institut für Polymerforschung, Ackermannweg 10, 55128 Mainz, Germany
}

(Received 10 March 2017; accepted 11 August 2017; published online 15 September 2017)

\begin{abstract}
Understanding the connections between the characteristic dynamical time scales associated with a coarse-grained (CG) and a detailed representation is central to the applicability of the coarse-graining methods to understand molecular processes. The process of coarse graining leads to an accelerated dynamics, owing to the smoothening of the underlying free-energy landscapes. Often a single timemapping factor is used to relate the time scales associated with the two representations. We critically examine this idea using a model system ideally suited for this purpose. Single molecular transport properties are studied via molecular dynamics simulations of the CG and atomistic representations of a liquid crystalline, azobenzene containing mesogen, simulated in the smectic and the isotropic phases. The out-of-plane dynamics in the smectic phase occurs via molecular hops from one smectic layer to the next. Hopping can occur via two mechanisms, with and without significant reorientation. The out-of-plane transport can be understood as a superposition of two (one associated with each mode of transport) independent continuous time random walks for which a single time-mapping factor would be rather inadequate. A comparison of the free-energy surfaces, relevant to the out-ofplane transport, qualitatively supports the above observations. Thus, this work underlines the need for building CG models that exhibit both structural and dynamical consistency to the underlying atomistic model. Published by AIP Publishing. [http://dx.doi.org/10.1063/1.5001482]
\end{abstract}

\section{INTRODUCTION}

While the connection between the length scales of simulation models with multiple levels of resolution ${ }^{1-3}$ is naturally provided by the mapping scheme (and a corresponding backmapping procedure), the link between the different time scales is not a priori known. Typically, coarse graining leads to accelerated dynamics due to smoother (free) energy landscapes compared to an all-atom description. Approaches to rigorously map dynamics of models on different scales or the methods to sample rare events ${ }^{4-7}$ are limited to simple model systems. The challenge in soft matter systems originates from the multitude of fluctuating free-energy barriers of comparable height. ${ }^{8-10}$ More pragmatic methods based on matching mean square displacements have been applied successfully to understand and predict dynamics in isotropic, one-component soft matter systems such as amorphous polymer melts, ${ }^{11-16}$ where the properties are dominated by the chain connectivity and non-crossability ${ }^{17-19}$ or the diffusion of additives ${ }^{20}$ in a polymer matrix. However, for more complicated scenarios such as multicomponent, anisotropic, or multiphase systems, the relationship between the dynamics in models at different resolution levels is by far not understood. For example, already in relatively simple two-component systems, the dynamics of a host polymer and smaller additive

\footnotetext{
a) Authors to whom correspondence should be addressed: biswaroop. mukherjee@gmail.com; christine.peter@uni-konstanz.de; and kremer@ mpip-mainz.mpg.de
}

molecules are accelerated very differently in a coarse-grained (CG) model. ${ }^{10}$ It has also been found that different dynamical modes in a single system (for example, translational diffusion compared to rotational diffusion or conformational transitions) can be affected differently upon coarse graining. ${ }^{21}$ It is this set of problems we want to address in the present publication, employing smectic liquid crystals (LCs) as ideal testing grounds.

Liquid crystals (LCs) are examples of materials which simultaneously exhibit the fluidity of a liquid phase and the type of ordering observed in solids. ${ }^{22,23}$ Though of huge technological relevance in the context of displays, they are also very valuable model systems from a more fundamental point of view. One reason for renewed interest in LC's is due to the wealth of different possible phases with a combination of orientational and full or partial translational order which can appear in these materials and which leads to richer phase diagrams compared to those of spherically isotropic particles. $^{24}$ This wealth of phases comes along with different, well-defined dynamical modes, making them ideal systems to study the effect of systematic coarse graining on the details of microscopic transport mechanisms.

Theoretically idealized models of LC molecules have been employed, both analytically ${ }^{25}$ and numerically. ${ }^{26,27}$ Despite their many advantages, they are unable to capture important features like the observed experimental dependence of the phase transition temperature on the molecular details, such as length or flexibility of the molecules. Though the atomistic models are capable of predicting details of the phases 
and the phase transitions such as the correct magnitudes of density change, ${ }^{28-33}$ some recently developed atomistic models of LC's also predict the transition temperatures very accurately. ${ }^{34-36}$ However, these atomistic models are often difficult to simulate over time scales long enough to ascertain the stability of the thermodynamic phases. That is the point where systematically coarse-grained models come into play, which can cover much longer length and time scales.

Recently we derived a CG model for a liquid crystalline compound with a rigid azobenzene mesogenic core and flexible tails ${ }^{37}$ [see Fig. 1, panel (a)]. The CG model correctly reproduces the structural properties of the smectic A (Sm-A) phase of $8 \mathrm{AB} 8$ at a temperature of $460 \mathrm{~K}$ as well as the isotropic to smectic transition near $480 \mathrm{~K}$. This model stays close to the atomistic system and retains chemical specificity and allows one to go back and forth between CG and atomistic resolutions using so-called backmapping techniques. ${ }^{38-40}$ A typical snapshot of the Sm-A phase is shown in Fig. 1 [panel (b)], where for reasons of clarity, only the $\mathrm{CG}$ beads belonging to the mesogenic core have been shown. This close proximity of the coarse-grained and the all-atom model makes this a perfect test case for coarse graining and dynamics, which is neither trivial nor too complicated since different modes of mesogen motion can clearly be identified in both representations. Details of the coarse-grained model and its parameterization are given in Ref. 37. The coarse-grained model accurately reproduces the phase transition, the jump in order parameter, and the small density jump of less than $1 \%$ at the LC/isotropic phase transition. The latter being in excellent agreement with experiments on molecular LC systems and in contrast to too large density changes of generic single site LC models of about $\sim 10 \%$. $^{41,42}$

Single molecule transport in anisotropic, liquid crystalline phases is an important area of research. From the technological

(a)

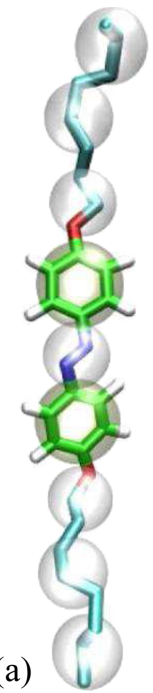

(b)

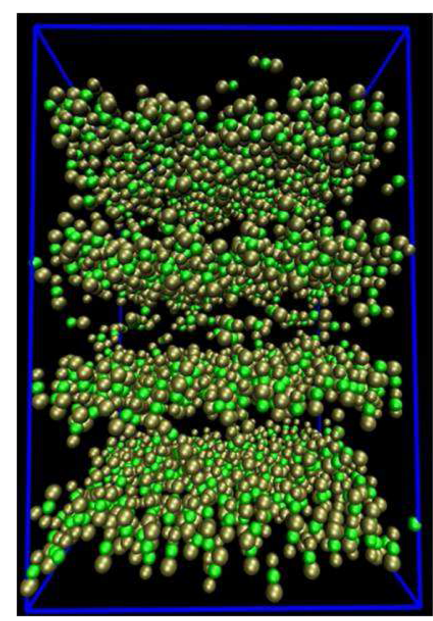

FIG. 1. (a) shows the CG representation, superimposed on the atomistic representation, of the azobenzene mesogens. (b) shows a typical snapshot of the smectic phase from the simulated trajectory. The four smectic layers are clearly visible along with the molecules located in the inter-layer spaces. For clarity, only the mesogenic core has been shown. aspect, understanding of diffusion in liquid crystalline environments is critical for improving the stability of LC display panels, ${ }^{43,44}$ whereas, from an academic perspective, the study of diffusion in the smectic phase helps one to uncover the intimate connection between the mesoscopic structure and the dynamics of constituent molecules. ${ }^{45}$ A particularly relevant class of dynamic modes in smectic systems are those related to the diffusion of mesogens both within the smectic layers and from layer to layer. It has already been established that inter-layer transport in smectics occurs via two different microscopic mechanisms. ${ }^{46}$ In the first, the straight mechanism, the translocation happens without significant reorientation and this mechanism has been observed in simulations of smectic phases formed by impenetrable spherocylinders ${ }^{47}$ and also in experiments with rigid viruses, self-assembled in a smectic phase. ${ }^{48,49}$ In the second mechanism, termed the parking lot (PKL) mechanism, the molecule moves from the initial layer to the next via an intermediate state where it is located in the space between the two smectic layers at an orientation which is perpendicular (transverse) to its initial and final directions. The parking lot mechanism takes advantage of the intrinsic flexibility of the molecules and is observed in both the all-atom and the coarse-grained simulations. ${ }^{46}$ This translocation mechanism occurs extremely rarely in simulations of the smectic phase using the generic, impenetrable spherocylinder models due to a high free-energy barrier $\left(\sim 20 \mathrm{k}_{B} \mathrm{~T}\right),{ }^{50,51}$ and thus the predominant translocation mechanism is the straight mechanism. In contrast, the typical translocation free-energy barriers encountered by $8 \mathrm{AB} 8$ molecules in the Sm-A phase in the $\mathrm{CG}$ simulations are approximately between 5 and $7 \mathrm{k}_{B} \mathrm{~T}^{46}$ for both mechanisms.

Rigorous methods of mapping dynamics in different but related models have originated from the attempt to accelerate infrequent transitions that are difficult to sample by using traditional molecular dynamics (MD) or MC methods. In hyperdynamics, accelerated transition events are introduced by modifying the original simulation Hamiltonian. This method ${ }^{4}$ was used to study diffusion in a 10-atom Ag cluster on the Ag (111) surface using a hyper-MD simulation of duration $\sim 200 \mu$ s. While these methods generally work well for lowdimensional potential energy surfaces (PES's), in situations with a large number of fluctuating degrees of freedom, the process of modifying the PES can become very cumbersome, if not impossible. The only polymer system where similar ideas have been applied is a melt of polyethylene (PE) chains, where this was applied to the torsion barriers along the backbone of the chains. ${ }^{7}$ Extension of this type of method to a different polymer such as polystyrene (PS) already led to huge difficulties. ${ }^{12,13}$

In several studies, the Hamiltonian dynamics on the $\mathrm{CG}$ potential energy surface has been replaced by a stochastic, Markovian dynamics ${ }^{52-60}$ (also non-Markovian dynamics in some cases ${ }^{61}$ ), which explicitly considers the effects of fluctuating frictional forces on the $\mathrm{CG}$ degrees of freedom. This friction stems from the degrees of freedom which have been integrated out. Their effect is then incorporated explicitly into the CG model so that the CG dynamics is on par with that observed for the atomistic description. In this context, several CG models are being derived with the aim of correctly reproducing 
the atomistic dynamics ${ }^{62-64}$ via incorporating fictitious particles in the CG model. These fictitious particle models, which are a special case of a particular class of models discussed by Zwanzig, ${ }^{65,66}$ interact with the CG degrees of freedom via non-linear interactions ${ }^{65}$ and are subject to Langevin forces. As a result, these fictitious particle CG models only affect the dynamics of the CG systems without modifying the underlying structure. These types of models are being developed for the multi-scale coarse-graining models ${ }^{67-71}$ and are being used to study the dynamics in simple systems like LennardJones fluids and dilute aqueous solutions of CG ethanol and liquid CG methanol. ${ }^{62-64}$ In principle, these methods can be applied to more complex systems, but this work remains to be done.

However, if one wants to link the intrinsic time scales of fine-grained and coarse-grained models for more complicated systems in a simple way, one has, so far, to resort to a more pragmatic approach. Typically mean squared displacements (MSDs, given by the formula, $\left\langle\Delta r^{2}(t)\right\rangle=\left\langle\left[\Delta \mathbf{r}_{i}\left(t_{0}, t_{0}+t\right)\right]^{2}\right\rangle$, where the angular brackets denote averaging over time-origins, $\mathrm{t}_{0}$, and the molecular index, i) from CG data, $\operatorname{MSD}\left(\mathrm{t}_{C G}\right)$, are shifted along the time-axis, $\operatorname{MSD}\left(\mathrm{st}_{C G}\right)$, until there is agreement with the atomistic data, $\operatorname{MSD}\left(\mathrm{t}_{A A}\right)$. This procedure is, however, applicable at time scales and distances where motion characteristics are similar in both atomistic and CG resolutions. This has been successfully used to obtain quantitative predictions of time scales from various CG simulations. ${ }^{11-16,72}$

Upon coarse graining and if one does not explicitly introduce additional frictional forces discussed above, the smoothening of energy landscape leads to accelerated dynamics. We would like to stress that in this work we do not introduce any additional frictional forces in the CG model and only consider the effects of dynamics on the smoother CG potential energy surface. To critically understand the various aspects of this accelerated dynamics, one should first identify the various competing microscopic mechanisms that combine to produce translational diffusion in the atomistic and the CG systems. With such knowledge, one should then monitor the speed-up of the individual processes and their relative probabilities of occurrences in the atomistic and CG descriptions and relate these to the properties of the corresponding free-energy surfaces. It is important to ascertain that the action of coarse graining has not introduced new microscopic pathways in the free-energy landscape. The task of identifying and classifying competing microscopic mechanisms is, in general, very complex. Here we can identify microscopic mechanisms, related to the out-of-plane transport, simultaneously in an atomistic and a CG representation of azobenzene containing LC molecules in the Sm-A phase. We then link these microscopic transport events to the macroscopic out-of-plane diffusion constant via a continuous time random walk (CTRW) formalism. ${ }^{73,74} \mathrm{Sim}$ ilar approaches have helped in understanding single-particle transport in several amorphous systems. ${ }^{75-84}$ This approach allows us to prove that the out-of-plane transport in the SmA phase is a superposition of two independent random walks (one for each type of translocation mechanism) with different rates, but with a single jump distance equal to the smectic layer spacing. These processes are then related to the free-energy landscapes in both the atomistic and the coarsegrained models, respectively.

The rest of the manuscript is arranged in the following way: in Sec. II we present the MD simulation procedure and the methods and algorithms which have been used to identify and characterize the various translocation mechanisms. We then determine the dynamics arising separately from each type of mechanism. In Sec. III these results are analyzed in detail and in Sec. IV we conclude.

\section{METHODS}

\section{A. Simulation details}

Both the atomistic and the CG simulations were performed with the GROMACS simulation package. ${ }^{85}$ For the atomistic simulations, a classical force-field, which is an adapted version of the (united atom) GROMOS $45 \mathrm{a} 3$ forcefield, was used, where the partial charges and the parameters of bonded interactions in the azobenzene and the alkoxy linker units have been re-parametrized based on quantum mechanical calculations discussed in Ref. 86. The derivation of the CG force-field has been carried out in a supercooled liquid phase $^{37}$ just below the smectic-isotropic phase transition. This supercooled phase is characterized by a high degree of local nematic order while being overall isotropic. With the resulting CG model, it is possible to switch between the atomistic and the CG resolutions (and vice versa) in a seamless manner maintaining values of all the relevant order parameters that describe the smectic A (Sm-A) state. The simulations of the Sm-A phase, in both resolutions, were carried out on a system of 1296 molecules arranged in four smectic layers. The $\mathrm{Z}$ direction of the simulation box was perpendicular to the smectic layers, i.e., parallel to the director. The atomistic simulations were performed for $400 \mathrm{~ns}$, with a simulation time step of $2 \mathrm{fs}$, and the snapshots were saved for further analysis every $100 \mathrm{ps}$. As in the atomistic simulations, the CG simulations were performed with masses considered in real units (amu, derived by summing all masses in the atomistic representation); energies appearing in the derived CG potentials are also considered in real units of $\mathrm{kJ} / \mathrm{mol}$. Similarly, length scales are considered in $\mathrm{nm}$, temperatures are in $\mathrm{K}$, and the coupling time of the thermostat is also in real time units of ps. Thus, the resulting time scales with the CG dynamics are also in real time units of $\mathrm{ps} / \mathrm{ns}$, up to dimensionless time-scaling factors originating due to the reduced microscopic friction in the CG representation. The values of these dimensionless time-scaling factors have been estimated for the different modes of single molecular transport in the Sm-A phase. The CG simulations were performed for a duration of $2000 \mathrm{~ns}$ in CG time units (a detailed discussion on the relation between atomistic and CG time scales is provided below) with a time step of $4 \mathrm{fs}$, and the snapshots were saved every 100 ps. Both the CG and the atomistic simulations were performed in the NVT ensemble and were thermostated with the global, canonical velocity rescaling thermostat ${ }^{87}$ with time-constant set to $0.2 \mathrm{ps}$. The effects of the thermostat and the chosen coupling time on the single molecule dynamics have been found to be negligible. A more detailed discussion on this aspect can be found in the supplementary material. 


\section{B. Analysis of dynamical modes}

All data analyses presented below have been performed on both the CG and the atomistic trajectories. First a spatial density distribution of the center of the molecules has been obtained as a function of the $\mathrm{z}$ coordinate using the formula

$$
\rho(z)=\left\langle\delta\left(z-z_{i}\right)\right\rangle
$$

where $\mathrm{z}_{i}$ is the $\mathrm{z}$ coordinate of the center of molecule "i" and the angular brackets denote averaging over both molecules and configurations. The four primary peaks of $\rho(\mathrm{z})$ in Fig. 2 occur at positions corresponding to the centers of the smectic layers where the upper panel of Fig. 2 shows the density distribution obtained from the CG simulations while the lower panel shows the same from the atomistic simulations. Upon comparing the distributions in the two resolutions, we observe that the peaks are sharper and taller in the CG description compared to the atomistic description where the distribution is more spread out. The inter-layer peaks in the CG description correspond to molecules residing in the spaces between two consecutive smectic layers in the CG simulations. This disparity results from the fact that the CG model was derived with the implicit aim of making it transferable
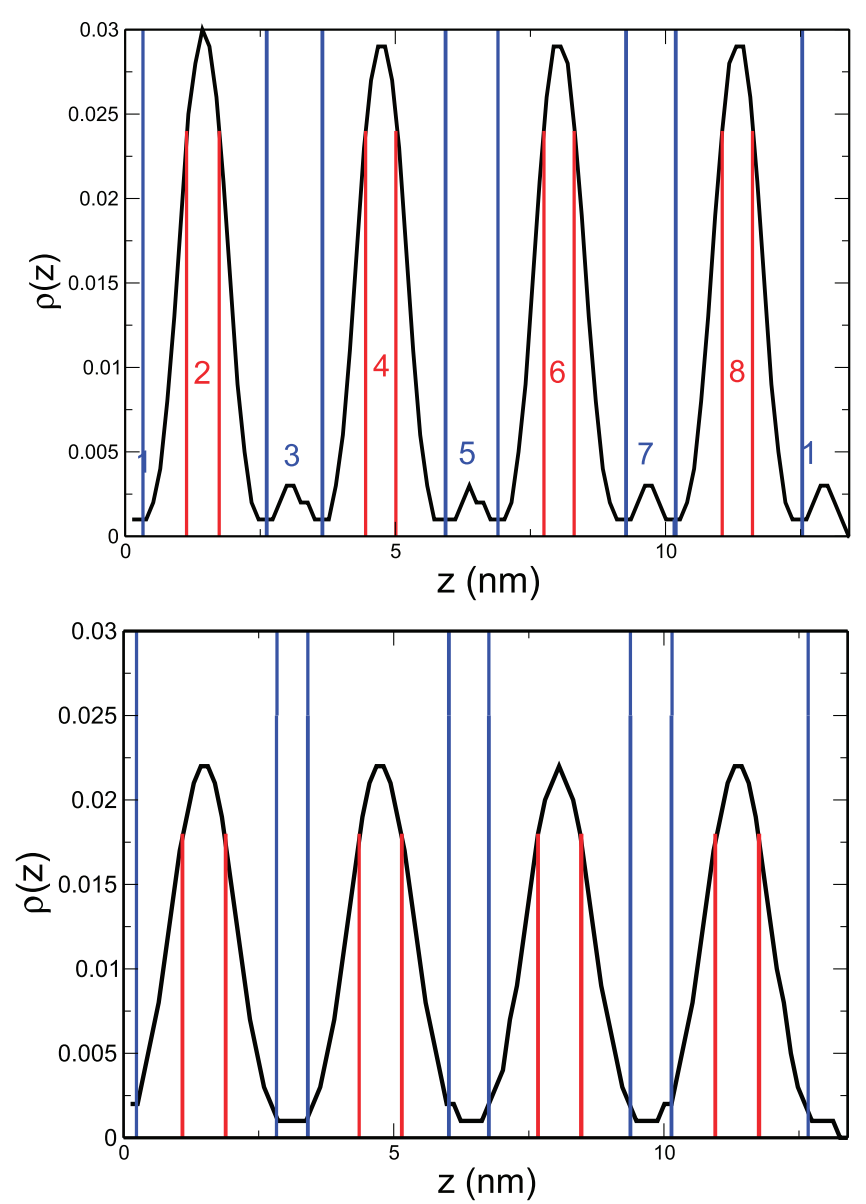

FIG. 2. The density of the centers of the LC molecules, $\rho(\mathrm{z})$, plotted as a function of the coordinate $\mathrm{z}$. The primary peaks correspond to the smectic layers and the secondary ones correspond to the location of the inter-layer molecules. The upper panel shows the CG density distribution, and the lower panel shows the atomistic counterpart. across an isotropic-smectic transition. Coarse graining was performed in the supercooled, isotropic phase at $460 \mathrm{~K}$, and these CG potentials were used to simulate the smectic phase. While the CG model derived via this approach was able to capture the broader details of isotropic-smectic phase transition, seemingly small differences showed up when one compared the free-energy surface (Fig. 15 of the present manuscript) relevant for the smectic phase in the two resolutions. This difference in the free-energy landscape leads to a difference in the details of the density distribution in the two representations.

In order to classify the trajectories (here we follow the $\mathrm{z}$ coordinate as a function of time as we are primarily interested in the out-of-plane dynamics of the LC molecules), each molecule is assigned an integer at every instant, which denotes where the molecule resides inside the simulation box. If the molecule resides inside a well-defined smectic layer, its z coordinate should fall within the two red vertical lines on either side of the primary peaks of $\rho(\mathrm{z})$. If this is the case, the molecule in question is assigned an "even" tag of either $2,4,6$, or 8 . The vertical red lines are drawn at values of the $\mathrm{Z}$ coordinate where the density values are $80 \%$ of the maximum. As a result, the exact locations of the smectic layers do not precisely coincide in the atomistic and the CG descriptions. Similarly if the z coordinate of the molecule is within the two vertical blue lines bracketing the secondary peaks, then it is identified as a molecule that is residing in the inter-layer space and it is assigned an "odd" tag of $1,3,5$, or 7 at that instant. A molecule is assigned a new tag only when it crosses from one well-defined region (either between two red lines or between two blue lines) to another, otherwise the molecule retains its old tag. As an example, if the molecule exits the region between two red lines (a given smectic layer), resides in the intermediate region, and returns back to the region between the region between the same set of red lines (the original smectic layer), the tag (discretised $\mathrm{Z}$ coordinate) retains its old value corresponding to that of the smectic layer. This exercise thus removes highfrequency oscillations present in the microscopic trajectories and thus helps reconstruct the out-of-plane dynamics based on the positional jumps corresponding to actual inter-layer transport events. The upper panel of Fig. 3(a) shows a real trajectory, whereas the middle panel shows the discretised version of the same trajectory. Once this has been performed for all molecules in the system, it is possible to identify all layer-to-layer translocation events from the time-series of the tag variable of each molecule. The middle panel of Fig. 3(a) shows the example of a parking lot translocation with the first discontinuity occurring at $t_{\text {start }}$ (time at which the center of the molecule moves from the initial smectic layer to the interlayer space) and the second one at $\mathrm{t}_{\text {end }}$ (time at which the center of the molecule again moves from the interlayer space back to a smectic layer).

So far we only considered the $\mathrm{z}$ coordinate of the center of the molecules. Since the LC molecules possess both translational and orientational degrees of freedom, one also has to monitor the angle each molecule makes with the director during the microscopic translocation processes discussed above. Figure 3(a) (lower panel) shows the time-series of the angle of the mesogenic core of the tagged molecule with the $\mathrm{Z}$ axis 

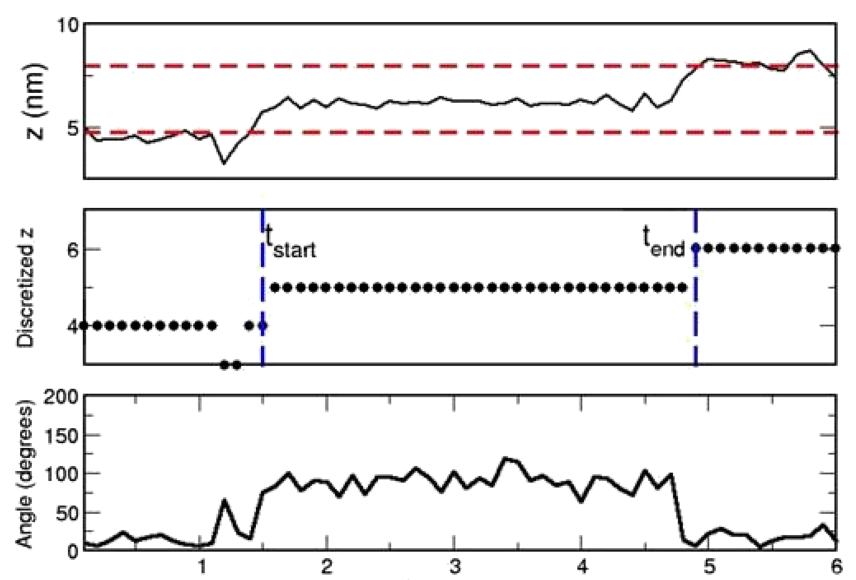

(a)

Time (ns)

(b)

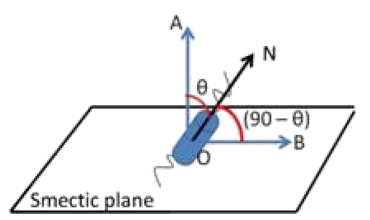

FIG. 3. A typical parking lot translocation event exhibited in terms of timeseries of three quantities is shown in (a). The upper panel of (a) shows the time-series of the $\mathrm{Z}$ coordinate of the chosen molecule during translocation. The broken horizontal lines correspond to the positions of the initial and the final Sm-A layers of the tagged molecule. The middle panel of (a) shows the time-series of the $\mathrm{Z}$ coordinate discretized via methods discussed in the text. The lower panel of (a) shows the time-series of the angle formed between the mesogenic core of the transiting molecule and the $\mathrm{Z}$ direction of the simulation box. It clearly shows that when the particle resides in the interlayer space (a value of five for the discretized $Z$ coordinate), its orientation is perpendicular to the $\mathrm{Z}$ direction (along the director), thus confirming that it is indeed a parking lot translocation. (b) shows the angle made by the axis of a chosen molecule with the $\mathrm{Z}$ axis of the simulation box, which coincides with the normal to the smectic plane.

of the simulation box. If during the translocation event $\left(\mathrm{t}_{\text {start }}\right.$ $\leq \mathrm{t} \leq \mathrm{t}_{\text {end }}$ ), the angle, $\theta$, between the axis of the molecule (N) and the director [see Fig. 3(b)] satisfies the inequality 190 $-\theta \mid \leq \theta_{\text {cutoff }}$, then the translocation event is identified as a parking lot (PKL) event, otherwise it is identified as a straight permeation event. This means that during the translocation if the angle between the axis of the tagged molecule $(\mathrm{N})$ and a direction along the smectic plane is less than $\theta_{\text {cutoff }}\left(\theta_{\text {cutoff }}\right.$ has been chosen to be $20^{\circ}$ ), then the translocation is identified as a parking lot translocation.

\section{Calculation of free-energy profile}

We have calculated the $2 \mathrm{D}$ free-energy profiles as a function of the position along the z-axis (i.e., perpendicular to the layers) and the angle formed by the mesogenic core with the $\mathrm{z}$ axis of the simulation box by the Boltzmann inversion of the respective $2 \mathrm{D}$ distribution functions. The $\mathrm{z}$ coordinate and the angle that the mesogenic core makes with the $\mathrm{z}$ axis were binned appropriately, taking into account the translational symmetry present in the $\mathrm{z}$ direction. The bin sizes for the translational and the orientational coordinates were $0.15 \mathrm{~nm}$ (layer spacing $\sim 3.3 \mathrm{~nm}$ ) and $\sim 3^{\circ}$, respectively. The free-energy landscape is then obtained by taking the negative logarithm of the $2 \mathrm{D}$ distribution function using the equation

$$
F(z, \theta)=-k_{B} T \ln [\pi(z, \theta)],
$$

where $\pi(z, \theta)$ is the $2 \mathrm{D}$ distribution function.

\section{RESULTS}

We first look at the diffusion of the molecules in the xy plane, where we do not distinguish between molecules located in the smectic layers or in the interlayer space. Figure 4 shows the in-plane MSD of the atomistic (broken line) and the unscaled CG simulations (solid line). Scaling the MSD by a shift factor $\left(s_{x y} \sim 3.5\right)$ (line with filled circles) leads to a perfect match of the curves. Thus, the dynamics perpendicular to the director is well described by a standard diffusion behavior down to the one nm scale. All the inter-molecular interactions average to an effective molecular friction. Figure 5 shows a similar estimation of the time-scaling factor by matching the in-plane MSD's for the continuously residing intra-layer (upper panel) and the inter-layer (lower panel) molecules. This time-scaling factor for both sub-ensembles of molecules is $\sim 3$, with the inter-layer molecules exhibiting faster in-plane dynamics compared to the intra-layer molecules. The intraand interlayer molecules experience a very different local environment. While the intra-layer molecules are primarily aligned along the director, the inter-layer molecules are oriented perpendicular to the director and are primarily surrounded by the alkyl tails of the neighbouring intra-layer molecules. In spite of this, the value of the time-scaling factor for the dynamics along the smectic planes is very similar for these two subensembles of molecules. This emphasizes that the reduction in local bead friction, upon transitioning from the atomistic to the CG model, does not strongly depend on the local environment (intra-layer versus inter-layer) the molecules find themselves in. The value of $s_{x y}$ is 3.0 for the intra-layer molecules, whereas, it is 3.1 for inter-layer molecules. The fact that these

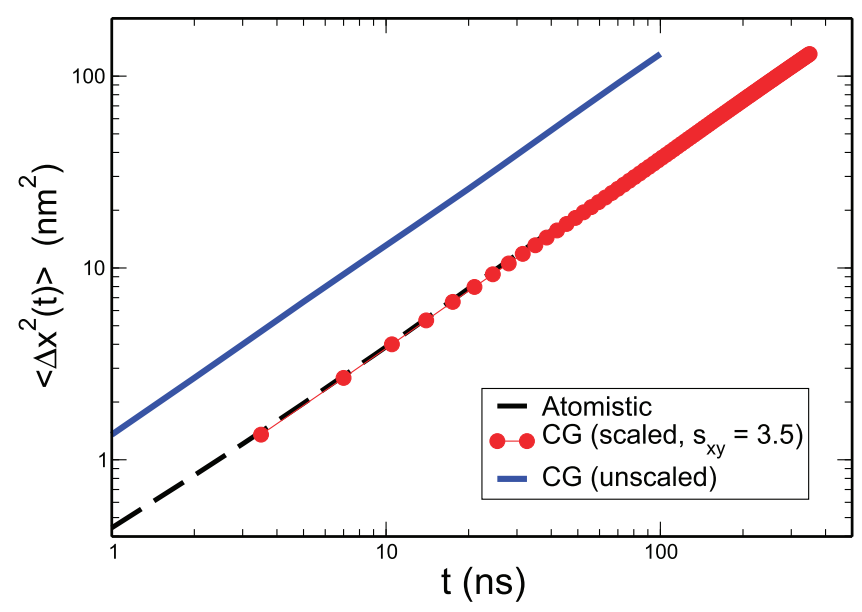

FIG. 4. MSD along the smectic planes from the atomistic (broken line) and the unscaled CG simulations (solid line). The scaled CG MSD (filled circles) is obtained from the unscaled MSD by shifting along the time axis till the data sets coincide. The proportionality constant that corresponds to the amount of shifting is the time-scaling factor $\left(\mathrm{s}_{x y} \sim 3.5\right)$ relating the intrinsic time scales of the atomistic and the CG simulations. This calculation has been performed over all molecules in the system, irrespective of whether they are within the Sm-A layer or they are residing in the inter-layer space. 

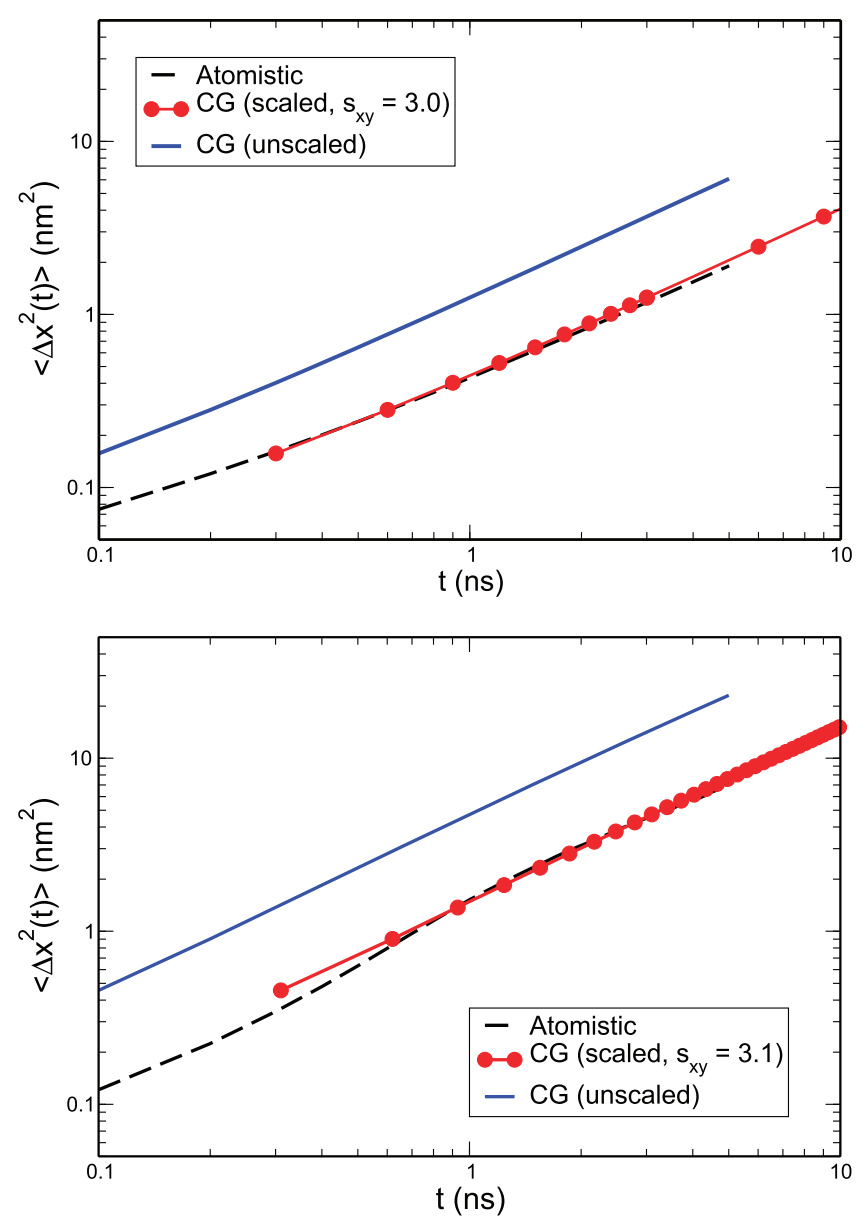

FIG. 5. The upper panel shows the estimation of the time-scaling factor by matching the in-plane MSD's for the molecules residing within the smectic layers. The lower panel shows the same for the in-plane MSD's of the molecules that reside in the inter-layer spaces. Both calculations have been performed till times where the molecules either stay continuously inside the smectic layers or reside in the inter-layer spaces.

time-scaling factors computed for the continuously residing inter- and intra-layer molecules are slightly different from the value of the time-scaling factor, $s_{x y} \sim 3.5$, which one obtains when one considers the in-plane dynamics of both inter- and intra-layer molecules (see Fig. 4), is interesting. This can be rationalised in the following way. Since the smectic phase consists of both inter- and intra-layer molecules, the average atomistic in-plane diffusion constant can be expressed as the weighted sum of the intra- and inter-layer diffusion constants via the expression $\left\langle D_{x y}^{a t m}\right\rangle \sim\left(n_{\text {inter }}^{\text {atm }} D_{x y-i n t e r}^{a t m}+n_{\text {intra }}^{\text {atm }} D_{x y-\text { intra }}^{a t m}\right)$. Here $\left\langle D_{x y}^{a t m}\right\rangle$ is the average atomistic in-plane diffusion constant, $D_{x y-i n t e r}^{a t m}$ is the atomistic in-plane diffusion constant of the inter-layer molecules, and $D_{x y-i n t r a}^{a t m}$ is the in-plane diffusion constant of the intra-layer molecules. $n_{\text {inter }}^{\text {atm }}$ is the fraction of inter-layer molecules and similarly $n_{\text {intra }}^{\text {atm }}$ is the fraction of intra-layer molecules in the atomistic simulations. Similarly the average $\mathrm{CG}$ in-plane diffusion constant can be expressed as $\left\langle D_{x y}^{C G}\right\rangle \sim\left(n_{\text {inter }}^{C G} D_{x y-\text { inter }}^{C G}+n_{\text {intra }}^{C G} D_{x y-\text { intra }}^{C G}\right)$, where the symbols have similar meanings as those for the corresponding atomistic expression. The value of the in-plane time-scaling factor, irrespective of the location of the molecules, is given by the expression $s_{x y} \sim\left\langle D_{x y}^{C G}\right\rangle /\left\langle D_{x y}^{a t m}\right\rangle$. Upon substituting the values $n_{\text {inter }}^{\text {atm }}=0.01, n_{\text {intra }}^{\text {atm }}=0.99, n_{\text {inter }}^{C G}=0.04, n_{\text {intra }}^{C G}=0.96$, and the appropriate values of the diffusion constants, we obtain $s_{x y} \sim 3.6$ from the ratio of the average in-plane diffusion constants, a value which matches with the value of $s_{x y}$ obtained from matching the in-plane MSD's in Fig. 4.

The out-of-plane dynamics represents a completely different scenario due to the smectic layers, which presents a permanent barrier for the molecules trying to diffuse from one smectic layer to its subsequent one. Three time scales dictate the out-of-plane dynamics. The most significant (and the longest) one is the residence time of the molecules within the smectic layers. These intra-layer residence times can be identified from Fig. 3 (middle panel) as the durations $\left(\mathrm{t} \leq \mathrm{t}_{\text {start }}\right)$ and $\left(\mathrm{t} \geq \mathrm{t}_{\text {end }}\right)$ when the tagged molecule resides in layers 4 and 6 , respectively. Next comes the inter-layer residence time which is the duration $\left(\mathrm{t}_{\text {start }} \leq \mathrm{t} \leq \mathrm{t}_{\text {end }}\right)$ during which the molecule resides in the inter-layer regions (see the middle panel of Fig. 3). The shortest time scale is identified as the time interval during which the tagged molecule transits from one smectic layer to its subsequent one (again see the middle panel of Fig. 3). The distributions of the residence times in the smectic layers are exponential and can be fitted with a single time scale, $\tau_{\text {res }}^{\text {layer }}$. Figure 6 presents the distribution of the residence times in the smectic layers for the atomistic and the CG simulations and their single-exponential fits yield $\tau_{\text {res, } C G}^{\text {layer }}=57.4 \mathrm{~ns}$ and $\tau_{\text {res,atm }}^{\text {layer }}=81 \mathrm{~ns}$. Similarly the distribution of residence times in the inter-layer region is shown in Fig. 7 (filled triangles) for the CG (upper panel) and atomistic (lower panel) simulations. These distributions are also exponential with characteristic time scales $\tau_{\text {res }, C G}^{\text {inter-layer }}=1.7 \mathrm{~ns}$ and $\tau_{\text {res,atm }}^{\text {inter-layer }}=1 \mathrm{~ns}$.

Of the two translocation mechanisms, the straight one is faster while the parking lot translocation process takes longer time and is made up of three steps, of which the first and the third are very short (see the positional jumps at $\mathrm{t}=\mathrm{t}_{\text {start }}$ and $\mathrm{t}$ $=\mathrm{t}_{\text {end }}$ in the middle panel of Fig. 3) with a translocation time $\sim 0.1 \mathrm{~ns}$ in atomistic time units, whereas the second portion is the most significant one. Figure 7 shows the distribution of the translocation times via the straight permeation (filled circles) and via the parking lot mechanism (filled squares) for

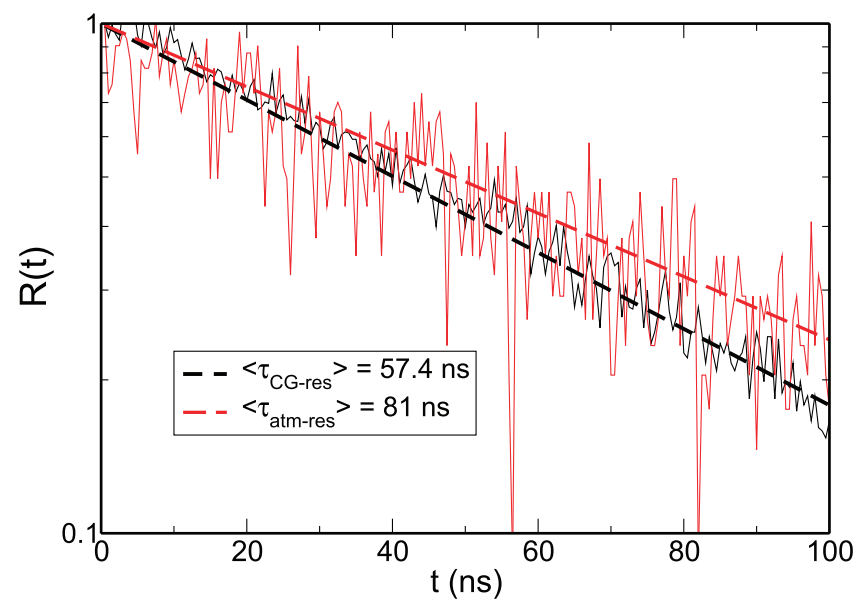

FIG. 6. The comparison of the distribution of the residence times in the smectic layers calculated from the atomistic and the CG simulations. These time scales are approximately inversely proportional to the out-of-plane diffusion constant. 

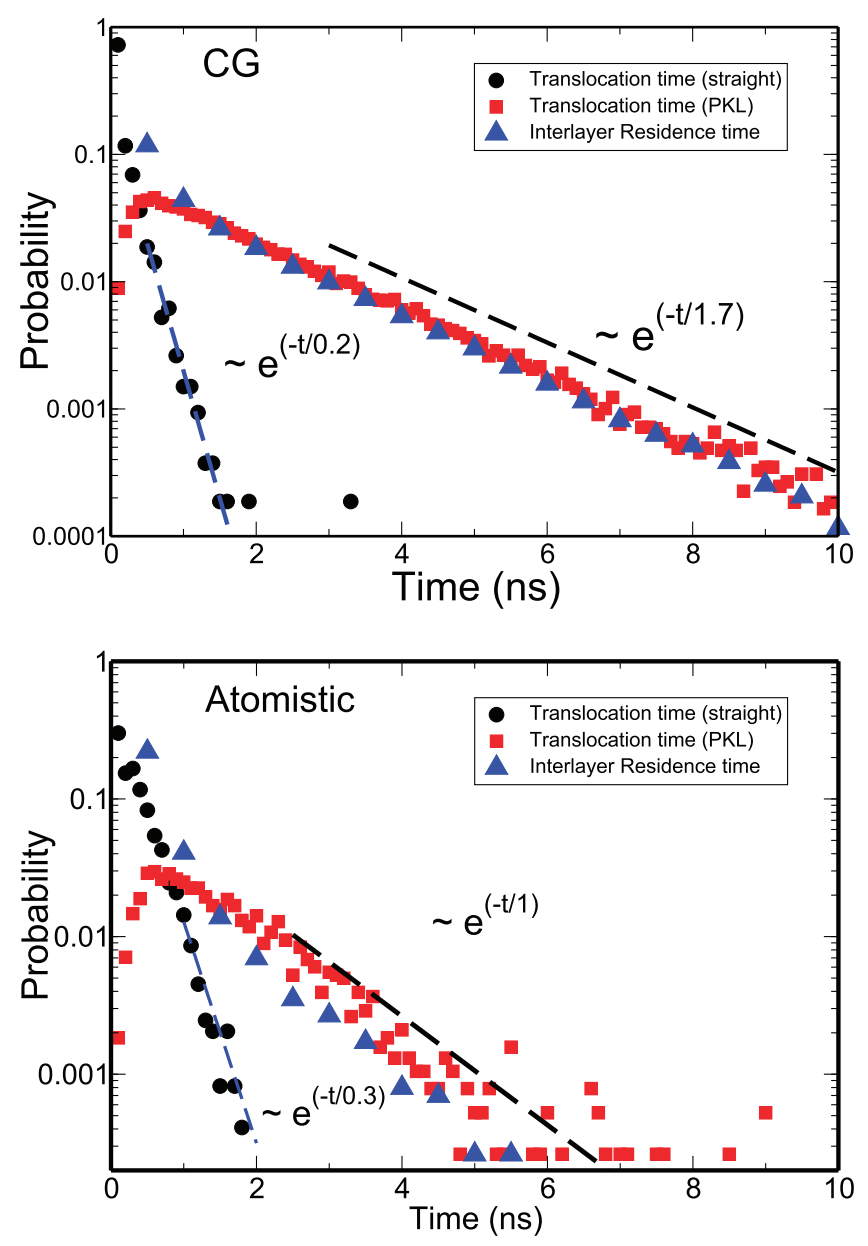

FIG. 7. The comparison of the distribution of the straight and the parking lot translocation times from one smectic layer to the subsequent ones and the distribution of the residence times in the interlayer space calculated for the CG (upper panel) and the atomistic (lower panel) simulations. The dashed lines are the fits of the distribution of the residence times via parking lot translocation.

CG (upper panel) and atomistic (lower panel) simulations. Since the residence time in the interlayer space dominates the behavior of the PKL translocation time, the distribution times for the whole process almost perfectly match that of the residence time. This holds for both the atomistic and the CG schemes. Table I provides a summary of the different time scales associated with the out-of-plane dynamics in the Sm-A phase. Interestingly, $\left\langle\tau_{\text {trans }}^{P K L}\right\rangle$ indicates that this process takes a much longer time compared to the straight translocation event for the $\mathrm{CG}$ simulations than for the AA simulations,

TABLE I. The summary of different time scales related to the out-of-plane motion in the $\mathrm{CG}$ and atomistic representations.

\begin{tabular}{lcc}
\hline \hline Time scales & $\mathrm{CG}($ unscaled) $(\mathrm{ns})$ & Atomistic $(\mathrm{ns})$ \\
\hline$\left\langle\tau_{\text {res }}^{\text {layer }}\right\rangle$ & 57.4 & 81 \\
$\left\langle\tau_{\text {trans }}^{\text {st }}\right\rangle$ & 0.2 & 0.3 \\
$\left\langle\tau_{\text {trans }}^{\text {pkl }}\right\rangle$ & 1.7 & 1.1 \\
$\left\langle\tau_{\text {res }}^{\text {inter-layer }}\right\rangle$ & 1.6 & 1 \\
$\left\langle\tau_{\text {st }}\right\rangle$ & 697 & 155 \\
$\left\langle\tau_{\text {pkl }}\right\rangle$ & 67 & 205 \\
$\left\langle\tau_{\text {eff }}\right\rangle$ & 61 & 88 \\
\hline \hline
\end{tabular}

indicating that either the relative probability to reside in the interlayer spacing is larger and/or that there are higher barriers to leave that region in qualitative agreement with the free-energy calculations (see below). Comparing all the relevant time scales in Table I characterizing the out-of-plane dynamics in the CG and the atomistic resolutions, it is obvious that a single time-mapping factor cannot be used to relate all the characteristic time scales of dynamics. A more general concept of a time-dependent time-mapping factor is more appropriate.

Figures 6 and 7 thus establish the fact that the out-ofplane microscopic dynamics is such that there are long periods of waiting punctuated by almost instantaneous jumps, during which molecules traverse distances comparable to their own length over very short durations. In a broader context, such intermittent nature of single molecule jumps appears to be a universal feature of dynamics in several model amorphous liquids. ${ }^{75-84}$ Understanding the long-time dynamics in terms of the statistical properties of these microscopic jumps using a CTRW framework has proved to be successful in several model systems that display glassy dynamics. ${ }^{75-78,82-84}$

Exponential distribution of waiting times in the smectic layers (see Fig. 6) signifies that the underlying process is Poissonian. ${ }^{88,89}$ For such a Poisson process with an exponential waiting time distribution,

$$
\psi(t)=\frac{1}{\langle\tau\rangle} e^{-t /\langle\tau\rangle}
$$

with the characteristic waiting time $\langle\tau\rangle$, it is possible to derive the probability, $\mathrm{P}(\mathrm{N}, \mathrm{t})$, of observing " $\mathrm{N}$ " translocations within a time interval "t," given by

$$
P(N, t)=\frac{(t /\langle\tau\rangle)^{N} e^{-t /\langle\tau\rangle}}{N !},
$$

where $\mathrm{P}(\mathrm{N}, \mathrm{t})$ is a Poisson distribution. From the above expression, one can evaluate the average number of translocations, $\langle N(t)\rangle$, within a time interval " $\mathrm{t}$ " via the relation

$$
\langle N(t)\rangle=\Sigma_{k=0}^{\infty} k P(k, t) .
$$

Upon substituting the expression for $\mathrm{P}(\mathrm{N}, \mathrm{t})$, one can show that the average number of translocations, $\langle N(t)\rangle$, occurring per molecule during a time interval $\mathrm{t}$ is given by

$$
\langle N(t)\rangle=t /\langle\tau\rangle
$$

showing that $\langle N(t)\rangle$ scales linearly with the time interval, $\mathrm{t}$, and inversely with the corresponding mean waiting time, $\langle\tau\rangle{ }^{88}$ In the present problem, however, there are two processes occurring in parallel (the straight and the parking lot translocations), and to understand the statistics of the number of translocation events in such a situation, we explicitly compute from the simulated trajectories the average number of total translocations, $\left\langle N_{\text {tot }}(t)\right\rangle$, the average number of straight translocations, $\left\langle N_{s t}(t)\right\rangle$, and the total number of parking lot translocations, $\left\langle N_{p k l}(t)\right\rangle$, during a time interval $t$. Microscopically the total number of translocations performed by a given molecule is equal to the sum of the number of straight and parking lot translocations. Such a relation should also hold for 
the corresponding averages, namely,

$$
\left\langle N_{t o t}(t)\right\rangle=\left\langle N_{s t}(t)\right\rangle+\left\langle N_{p k l}(t)\right\rangle .
$$

Figure 8 shows the time dependence of $\left\langle N_{\text {tot }}(t)\right\rangle,\left\langle N_{s t}(t)\right\rangle$, and $\left\langle N_{p k l}(t)\right\rangle$ computed from the CG (upper panel) and the atomistic simulations (lower panel). It is evident that at long times, all the three average quantities exhibit a linear dependence on the time interval, t [see Eq. (6)]. This establishes that the simulated out-of-plane dynamics can be approximated as a superposition of two independent Poisson processes and thus one can extract three time scales. Fitting $\left\langle N_{\text {tot }}(t)\right\rangle$ to $t /\left\langle\tau_{\text {eff }}\right\rangle$, $\left\langle N_{p k l}(t)\right\rangle$ to $t /\left\langle\tau_{p k l}\right\rangle$, and $\left\langle N_{s t}(t)\right\rangle$ to $t /\left\langle\tau_{s t}\right\rangle$, we extract these time scales from the analyses of the CG and atomistic trajectories. Here $\left\langle\tau_{\text {eff }}\right\rangle$ is identified as the average waiting time between two consecutive translocation events of an arbitrarily chosen molecule. Similarly $\left\langle\tau_{s t}\right\rangle$ is the average waiting time between two consecutive straight translocations and $\left\langle\tau_{p k l}\right\rangle$ is similarly the average waiting time between two consecutive parking lot translocation events. These values are listed in Table I. Using Eq. (7), we can relate the three time scales in each description via the relation

$$
\frac{1}{\left\langle\tau_{e f f}\right\rangle}=\frac{1}{\left\langle\tau_{s t}\right\rangle}+\frac{1}{\left\langle\tau_{p k l}\right\rangle}
$$
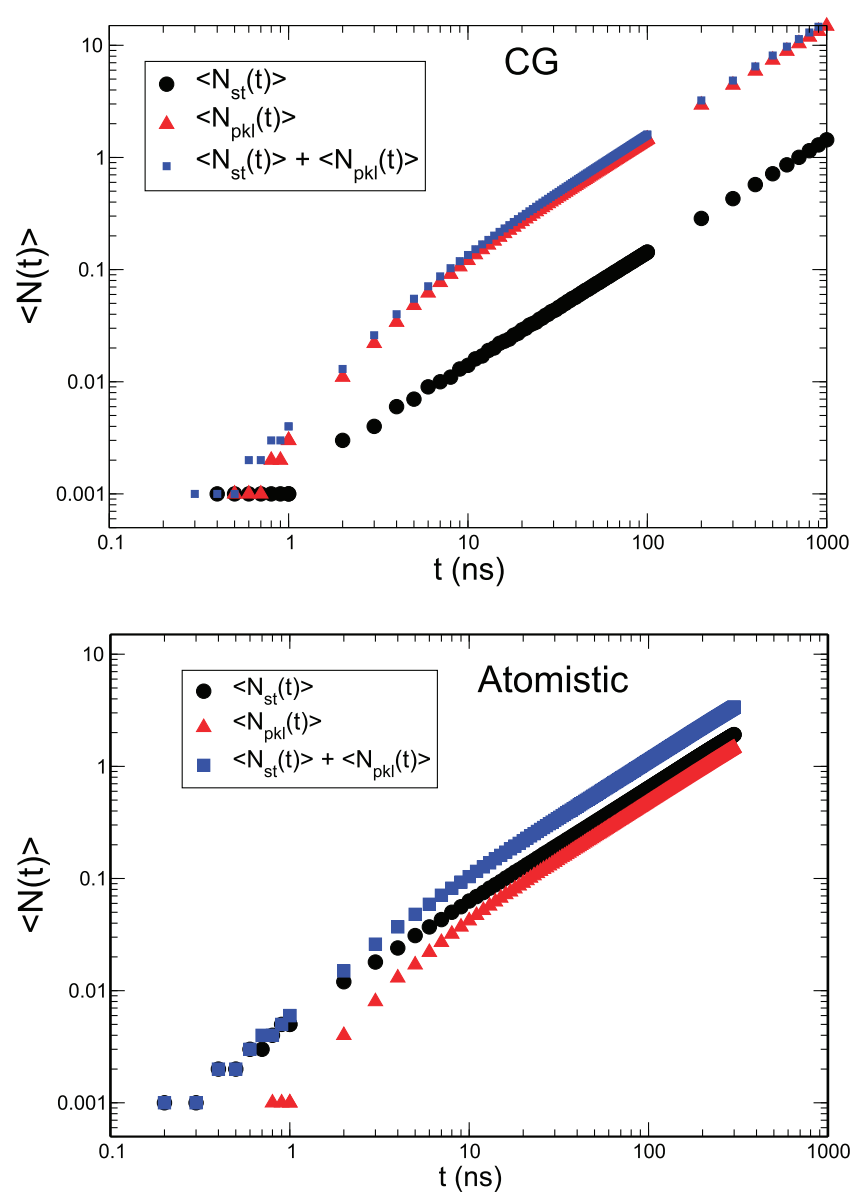

FIG. 8. The time-evolution of $\left\langle N_{s t}(t)\right\rangle,\left\langle N_{p k l}(t)\right\rangle$, and $\left\langle N_{\text {tot }}(t)\right\rangle\left(\left\langle N_{\text {tot }}(t)\right\rangle\right.$ $\left.=\left\langle N_{s t}(t)\right\rangle+\left\langle N_{p k l}(t)\right\rangle\right)$, computed from the CG simulations (upper panel) and the atomistic simulations (lower panel).
It is expected that $\left\langle\tau_{\text {eff }}\right\rangle$ should be close to the independently calculated mean waiting time in the smectic layers, from the distribution of the waiting times presented earlier. By comparing the values of the time scales, $\left\langle\tau_{\text {eff }}\right\rangle$ and $\left\langle\tau_{\text {res }}\right\rangle$, in Table I, it turns out that mean waiting times computed via two different routes agree with each other.

Figure 9 shows that the out-of-plane MSD computed from the simulated CG (upper panel) and the atomistic (lower panel) trajectories can be related to the average number of total translocation events, $\left\langle N_{t o t}(t)\right\rangle$, during time $t$, via the relation

$$
\left\langle\Delta z^{2}(t)\right\rangle=l_{s m}^{2}\left\langle N_{t o t}(t)\right\rangle
$$

for both the CG and the atomistic simulations as the molecules undergo a displacement of magnitude $l_{s m}$ during each translocation event. The inset shows the time dependence of the average number of total translocation events, $\left\langle N_{t o t}(t)\right\rangle$. The dashed line in the main plots show that $l_{s m}^{2}\left\langle N_{t o t}(t)\right\rangle$ exactly coincides with the out-of-plane MSD, $\left\langle\Delta z^{2}(t)\right\rangle$, at long times. Therefore, in the diffusive regime, $\left\langle\Delta z^{2}(t)\right\rangle$ can be written as a superposition of two CTRW's,

$$
\begin{aligned}
\left\langle\Delta z^{2}(t)\right\rangle & =l_{s m}^{2} \frac{t}{\left\langle\tau_{\text {eff }}\right\rangle} \\
& =l_{s m}^{2} t\left[\frac{1}{\left\langle\tau_{s t}\right\rangle}+\frac{1}{\left\langle\tau_{p k l}\right\rangle}\right],
\end{aligned}
$$
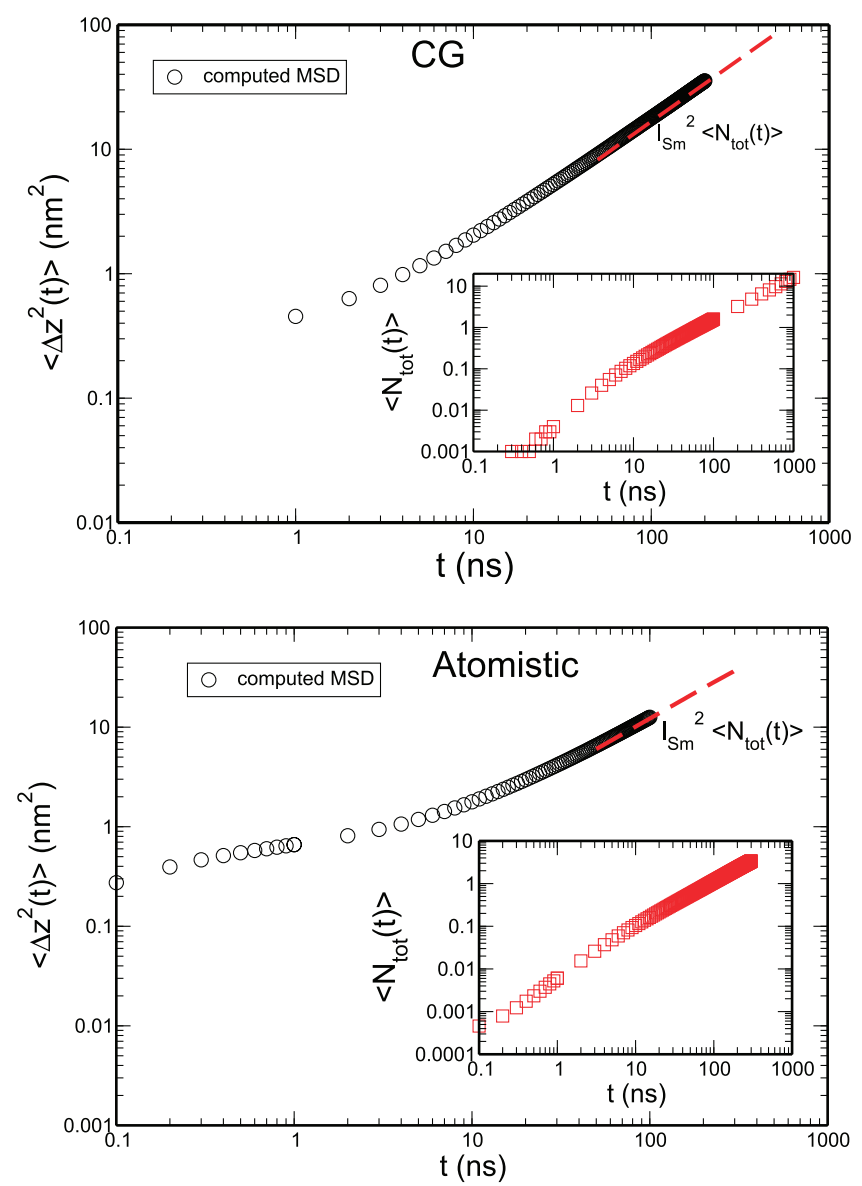

FIG. 9. The total out-of-plane MSD (circles) in the CG description (upper panel) and the atomistic description (lower panel), related to the average number of total steps in the out-of-plane direction (squares). The insets show the time dependence of $\left\langle N_{t o t}(t)\right\rangle$. 


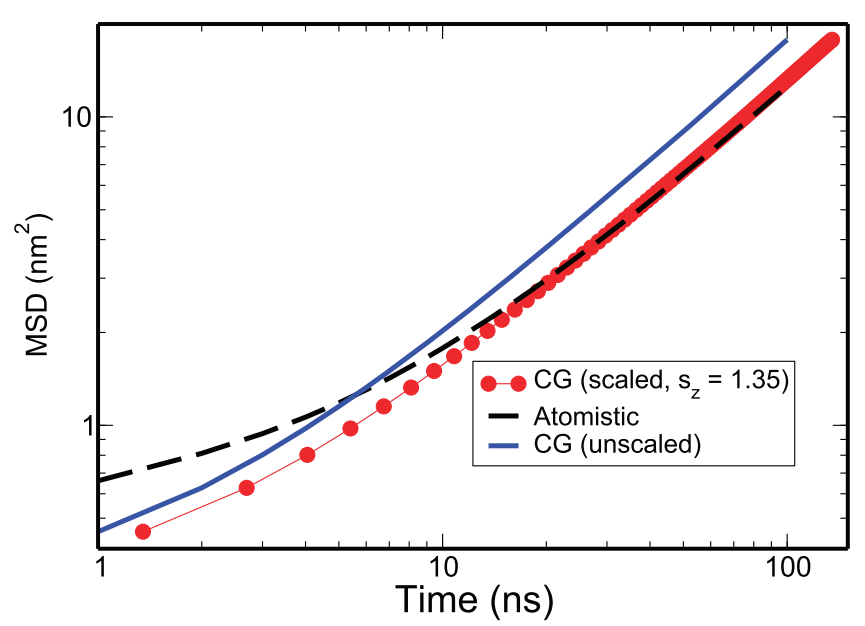

FIG. 10. The out-of-plane MSD obtained from the atomistic (broken line) and the unscaled CG simulation (solid line). The scaled CG MSD (filled circles) is again obtained from the unscaled MSD by shifting it along the time axis till the CG MSD coincides with the atomistic one. The proportionality constant that corresponds to the amount of shift is the time-scaling factor $\left(\mathrm{s}_{z} \sim 1.35\right)$ relating the intrinsic time scales of the atomistic and the CG dynamics in the out-of-plane direction. As in Fig. 4, the calculation has been performed over all molecules in the system, irrespective of whether they are within the Sm-A layer or they are residing in the inter-layer space.

with characteristic waiting times, $\left\langle\tau_{s t}\right\rangle$ and $\left\langle\tau_{p k l}\right\rangle$, and similar jump lengths. Figure 10 shows the time-scaling factor associated with the out-of-plane dynamics by matching the atomistic and the CG MSD's. The relevant time-scaling factor, $s_{z}$, turns out to be $\sim 1.35$, a value close to the ratios $(\sim 1.4$, see Table I) of $\left\langle\tau_{\text {res }}^{\text {layer }}\right\rangle$ and $\left\langle\tau_{\text {eff }}\right\rangle$ calculated from the atomistic and the CG trajectories.

Since the constituent processes are Poissonian [see Eq. (4)], they follow the relations

$$
P_{s t}\left(N_{s t}, t\right)=\frac{\left(\frac{t}{\left\langle\tau_{s t}\right\rangle}\right)^{N_{s t}} e^{-t /\left\langle\tau_{s t}\right\rangle}}{N_{s t} !}
$$

and

$$
P_{p k l}\left(N_{p k l}, t\right)=\frac{\left(\frac{t}{\left\langle\tau_{p k l}\right\rangle}\right)^{N_{p k l}} e^{-t /\left\langle\tau_{p k l}\right\rangle}}{N_{p k l} !} .
$$

Since these are independent, the probability distribution of the total number of steps is given by

$$
P_{t o t}\left(N_{t o t}, t\right)=\Sigma_{N_{s t}=0}^{N_{t o t}} P_{s t}\left(N_{s t}, t\right) P_{p k l}\left(\left(N_{t o t}-N_{s t}\right), t\right),
$$

which can be reduced to

$$
P_{t o t}\left(N_{t o t}, t\right)=\frac{\left(\frac{t}{\left\langle\tau_{e f f}\right\rangle}\right)^{N_{t o t}} e^{-t /\left\langle\tau_{e f f}\right\rangle}}{N_{t o t} !} .
$$

These quantities can be explicitly computed from the simulated trajectories and directly compared with those predicted by the CTRW approach [see Eq. (14)] with appropriate values of $\tau_{\text {eff }}$, which turn out to be $61 \mathrm{~ns}$ for the CG simulations and $88 \mathrm{~ns}$ for the atomistic simulations. Figure 11 shows the timeevolution of $P_{t o t}\left(N_{t o t}, t\right)$ computed from the CG simulations (line) and the expression in Eq. (14) (circles) at four values of time interval, t. Figure 12 shows a similar calculation obtained from atomistic simulations. At short times, these distributions are peaked at $N_{\text {tot }}=0$, which signifies that most molecules have not performed any translocations. At long times, $P_{t o t}\left(N_{t o t}, t\right)$ becomes a Gaussian peaked at non-zero values of $N_{t o t}$. The above results clearly establish that the statistics of the outof-plane translocation events, for both the atomistic and CG descriptions, can be viewed as a superposition of two independent random walks with different waiting times but similar jump lengths.

Neutron scattering experiments probe the dynamics in liquids by measuring its dynamic structure factor. ${ }^{90}$ The dynamic structure factor is related through a space-time Fourier transform to $\mathrm{G}_{s}(\mathbf{r}, t)$, the auto-correlation of number density at two different space-time points in the liquid. ${ }^{91} \mathrm{G}_{s}(\mathbf{r}, t)$ is identified as the self-part of the Van Hove correlation function $(\mathrm{VHCF})^{92}$
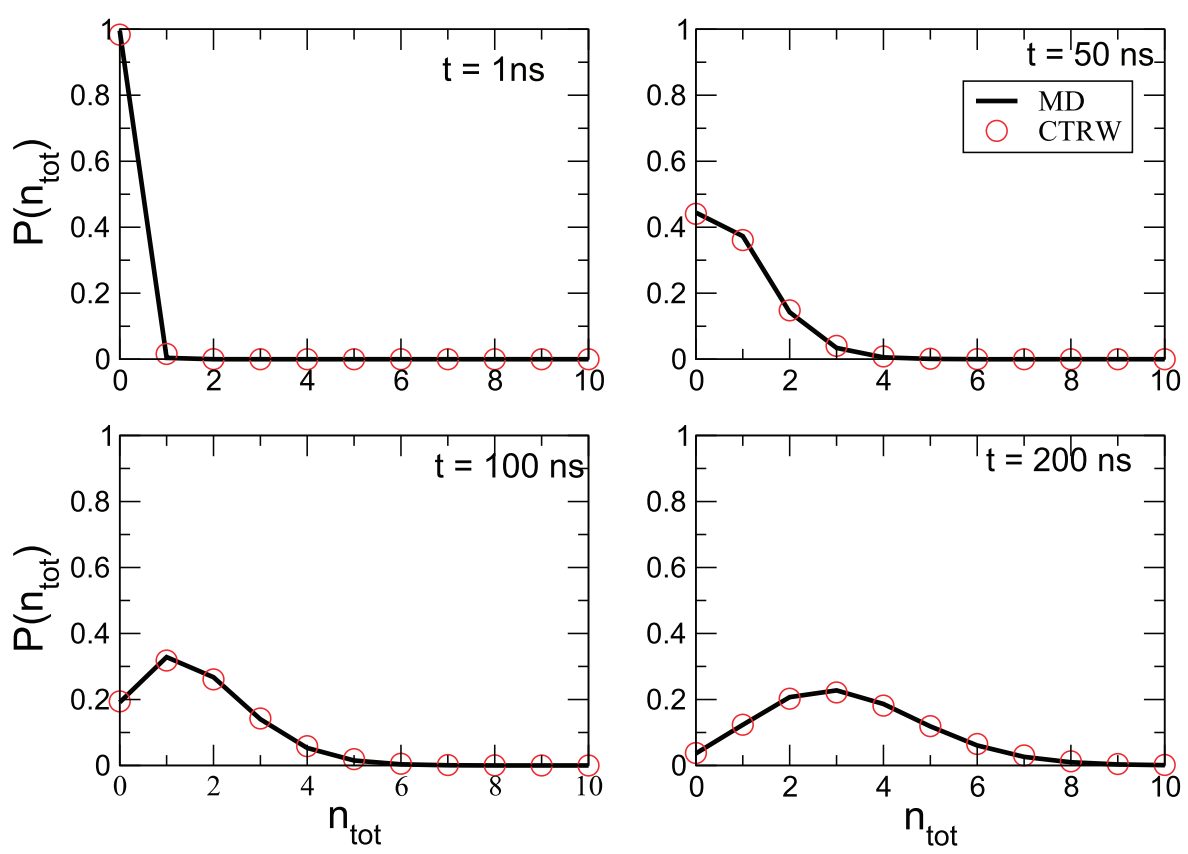

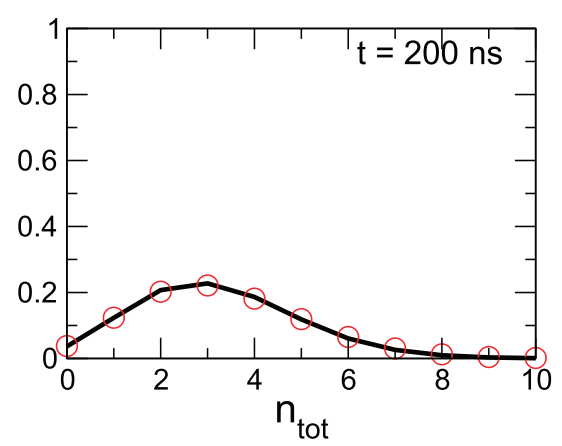

FIG. 11. The probability distribution of $N_{\text {tot }}$ jumps in time interval t, computed directly from the CG MD trajectories and the random walk model with the appropriate $\left\langle\tau_{e f f}\right\rangle=61 \mathrm{~ns}$, is plotted for the CG simulations. 

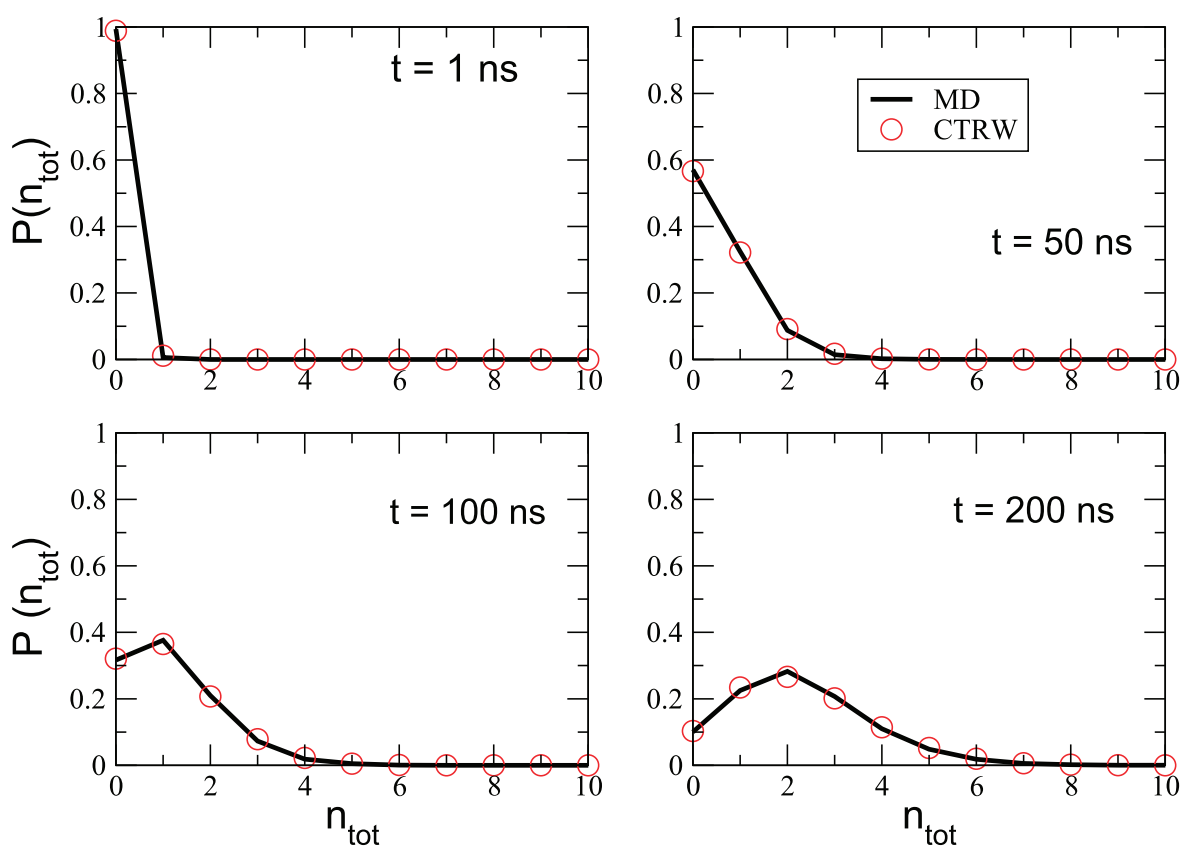

FIG. 12. The probability distribution of $N_{\text {tot }}$ jumps in time interval t, computed directly from the atomistic MD trajectories and the random walk model with the appropriate $\left\langle\tau_{\text {eff }}\right\rangle=88 \mathrm{~ns}$, is plotted for the atomistic simulations. defined via the relation

$$
G_{s}(\mathbf{r}, t)=\frac{1}{N}\left\langle\sum_{i=1}^{N} \delta\left(\mathbf{r}-\left[\mathbf{r}_{\mathbf{i}}\left(t_{0}+t\right)-\mathbf{r}_{\mathbf{i}}\left(t_{0}\right)\right]\right)\right\rangle_{t_{0}},
$$

where $\mathbf{r}$ is the displacement undergone in the time interval of duration $t$. The averaging is performed over both timeorigins, $t_{0}$, and the molecules (denoted by $i$ ) in the system. Thus, $\mathrm{G}_{s}(\mathbf{r}, t)$ measures the probability of observing a tagged molecule at position $\mathbf{r}$ at a time $\left(\mathrm{t}_{0}+\mathrm{t}\right)$, provided it was located at the origin of coordinates at a time $t_{0}$, which is an arbitrary time-origin. It can be readily computed from MD trajectories using the above relation. Here we consider the onedimensional version, $\mathrm{G}_{s}(\mathrm{z}, \mathrm{t})$, where $\mathrm{z}$ denotes displacements along the out-of-plane directions in the smectic phase. The CTRW formalism ${ }^{74-77}$ provides an alternative way to express the $\mathrm{VHCF}, \mathrm{G}_{s}(z, t)$, using the relation

$$
G_{s}(z, t)=\sum_{N_{t o t}=0}^{\infty} g\left(N_{t o t}, z\right) P\left(N_{t o t}, t\right),
$$

where $\mathrm{g}\left(\mathrm{N}_{t o t}, \mathbf{r}\right)$ is the probability that the molecule undergoes a displacement $\mathbf{r}$ in $\mathrm{N}_{\text {tot }}$ jumps and $\mathrm{P}\left(\mathrm{N}_{t o t}, \mathrm{t}\right)$ is similarly the probability that the molecule undergoes $\mathrm{N}_{\text {tot }}$ jumps during time $t$. In this paper, we present a discrete version of the VHCF, $\mathrm{G}_{s}(m, t)$, where the out-of-plane displacement ( $\mathrm{z}=\mathrm{m} 1_{s m}, \mathrm{~m}$ is an integer) assumes values that are only integral multiples of the smectic layer spacing. This CTRW description does not consider the effects of local vibrations and only the successful layer-to-layer translocation events are included. Thus, the discretized VHCF obeys the following relation:

$$
G_{s}(m, t)=\sum_{N_{\text {tot }}=0}^{\infty} g\left(N_{\text {tot }}, m\right) P\left(N_{t o t}, t\right) .
$$

For the purpose of the present manuscript, $\mathrm{g}\left(\mathrm{N}_{t o t}, \mathrm{~m}\right)$ and $\mathrm{P}\left(\mathrm{N}_{\text {tot }}, \mathrm{t}\right)$ have been obtained from the analysis of the $\mathrm{CG}$ and the atomistic MD trajectories and it has already been shown that $\mathrm{P}\left(\mathrm{N}_{t o t}, \mathrm{t}\right)$ can be described by a Poisson distribution with an appropriate time scale, $\tau_{\text {eff }}$. Figure 13 shows the comparison of the time-evolution of the discretized $\mathrm{G}_{s}(m, t)$ (red bars), which has been computed from the CTRW model using Eq. (17), and the continuous $\mathrm{G}_{s}(z, t)$ (black line), computed directly from the MD trajectories using Eq. (15), for the CG simulations. A similar comparison is also presented for the atomistic simulations in Fig. 14. The agreement between the results obtained via two routes again establishes that the out-of-plane dynamics comprising a superposition of two independent random walks is a very good approximation of the simulated CG and atomistic dynamics.

Representing the out-of-plane dynamics as a superposition of independent CTRW's allows us to estimate the amounts by which each individual translocation process speeds up when we move from an atomistic to the CG description. The values of the various time scales associated with the out-of-plane dynamics are presented in Table I and they clearly show that the speed-up upon coarse graining, compared to the atomistic system, is different for the two translocation mechanisms. From the comparison of the average number of total translocation events, $\left\langle N_{t o t}(t)\right\rangle$, the average number of straight translocations, $\left\langle N_{s t}(t)\right\rangle$, and the total number of parking lot translocations, $\left\langle N_{p k l}(t)\right\rangle$, during a time interval $t$, in Fig. 8 and the corresponding time scales in Table I, it is clear that the out-ofplane dynamics in the CG model, unlike the atomistic one, is dominated by parking lot translocation events. Since $\left\langle N_{p k l}(t)\right\rangle$ $\sim t /\left\langle\tau_{p k l}\right\rangle$ and $\left\langle N_{s t}(t)\right\rangle \sim t /\left\langle\tau_{s t}\right\rangle$, the relative fractions of the parking lot $\left(\mathrm{f}_{p k l}\right)$ and straight $\left(\mathrm{f}_{s t}\right)$ translocations are $f_{p k l} \sim \frac{\left\langle\tau_{e f f}\right\rangle}{\left\langle\tau_{p k l}\right\rangle}$ and $f_{s t} \sim \frac{\left\langle\tau_{e f f}\right\rangle}{\left\langle\tau_{s t}\right\rangle}$, respectively. Thus, $f_{p k l}$ is $\sim 0.9$ in the CG simulations, whereas it is $\sim 0.43$ in the atomistic simulations. Although the CG model, parametrized in the supercooled, isotropic phase at $460 \mathrm{~K}$, perfectly reproduces the isotropic to Sm-A phase transition and the associated jump in the order parameter and the density discontinuity, the relative weights of the constituent microscopic processes are not maintained in 

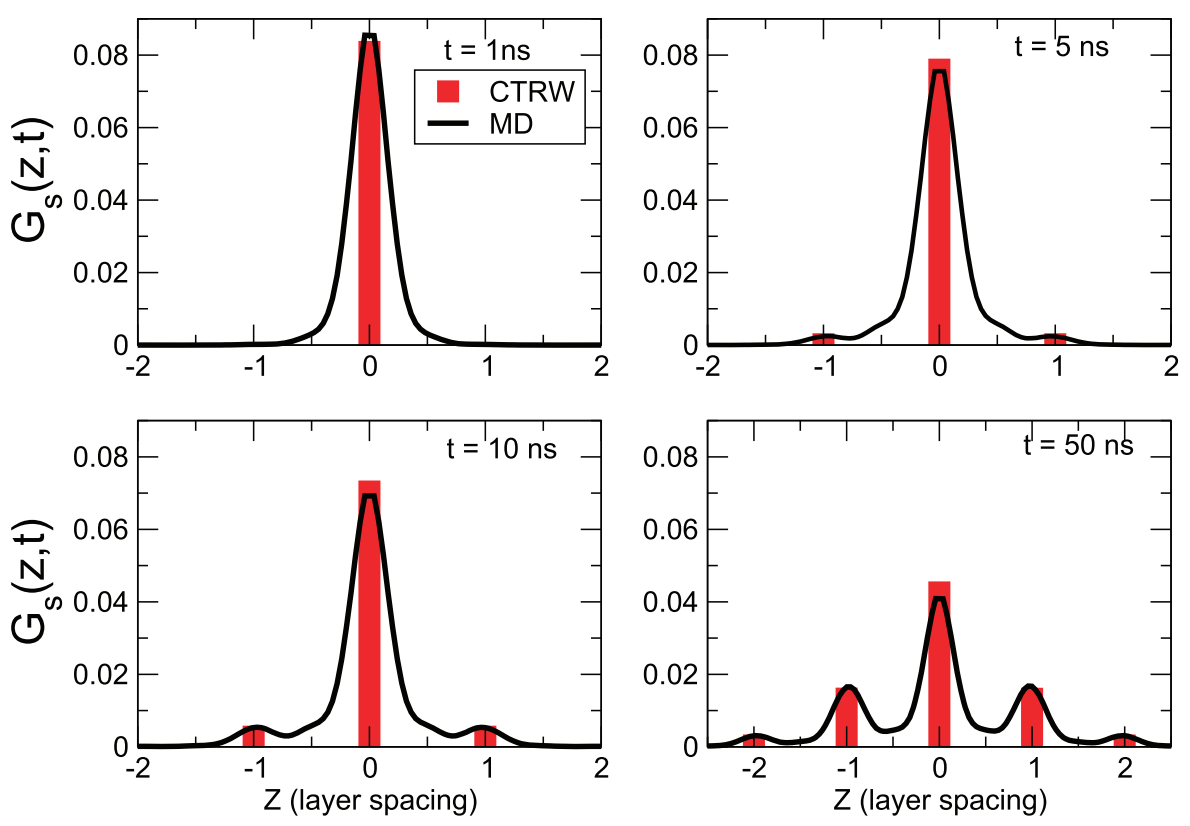

FIG. 13. The self-part of the VHCF, $\mathrm{G}_{s}(\mathrm{z}, \mathrm{t})$, computed from the CG MD trajectories and those computed from the discrete version of the CTRW model compared at several times. the accelerated CG dynamics. Figure 15 shows the comparison of the CG (upper panel) and the atomistic (lower panel) free-energy surfaces. Stable minima occur at the bottom and top left of the surfaces, which represent a molecule sitting at the center of a Sm-A layer with its axis aligned with the director. An additional local minimum at half layer spacing with $\theta=90^{\circ}$ originates from the presence of the transverse, interlayer molecules. Although this feature is also present in the atomistic free-energy surface (upper panel of Fig. 15), it is more prominent for the CG free-energy surface (upper panel of Fig. 15). This explains the high percentage of parking lot processes and the long residence times in the interlayer spaces observed in the CG simulations. This is in qualitative agreement to various values of the time scales associated with the out-of-plane motion in the CG and atomistic descriptions provided in Table I. In our earlier publication, we have already shown that the typical CG free-energy barrier, owing to the internal flexibility of the molecules, was typically between 5 and $7 \mathrm{k}_{B} \mathrm{~T}$ along any arbitrarily chosen straight and parking lot translocations. ${ }^{46}$ The situation, however, is very different for the Sm-A phase formed by hard spherocylinders, where the free-energy penalty for the transverse, interlayer state was estimated to be $\sim 20 k_{B} T ;{ }^{51}$ hence, transverse, interlayer molecules were rarely observed in these simulations and the straight hops between subsequent layers were the only relevant mode of translocation.

The different translocation schemes are closely connected to the ability of the molecules to rotate against the director axis. While this is almost impossible for hard rods, the present molecules are more flexible due to the presence of aliphatic tails. It is thus interesting to check whether one can also observe similar signatures already in the
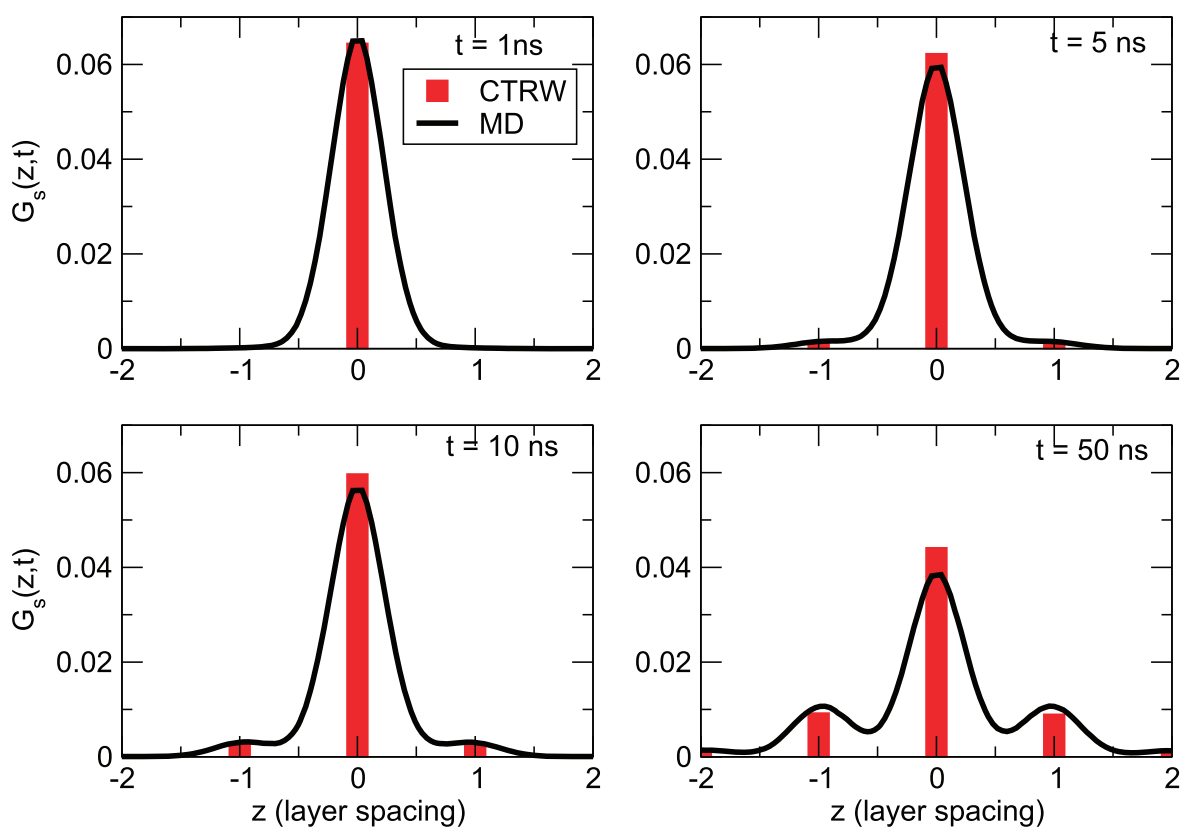

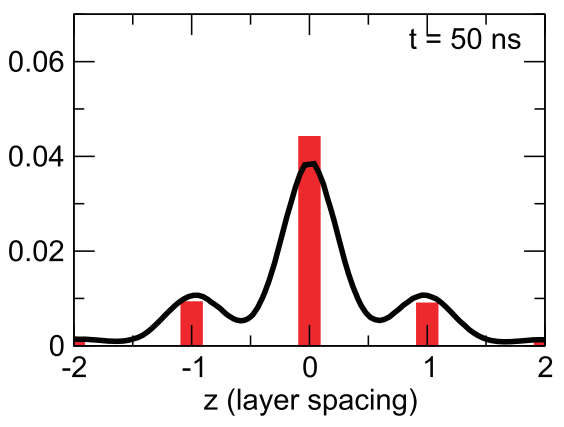

FIG. 14. The self-part of the VHCF, $\mathrm{G}_{s}(\mathrm{z}, \mathrm{t})$, computed from the atomistic MD trajectories and those computed from the discrete version of the CTRW model compared at several times. 

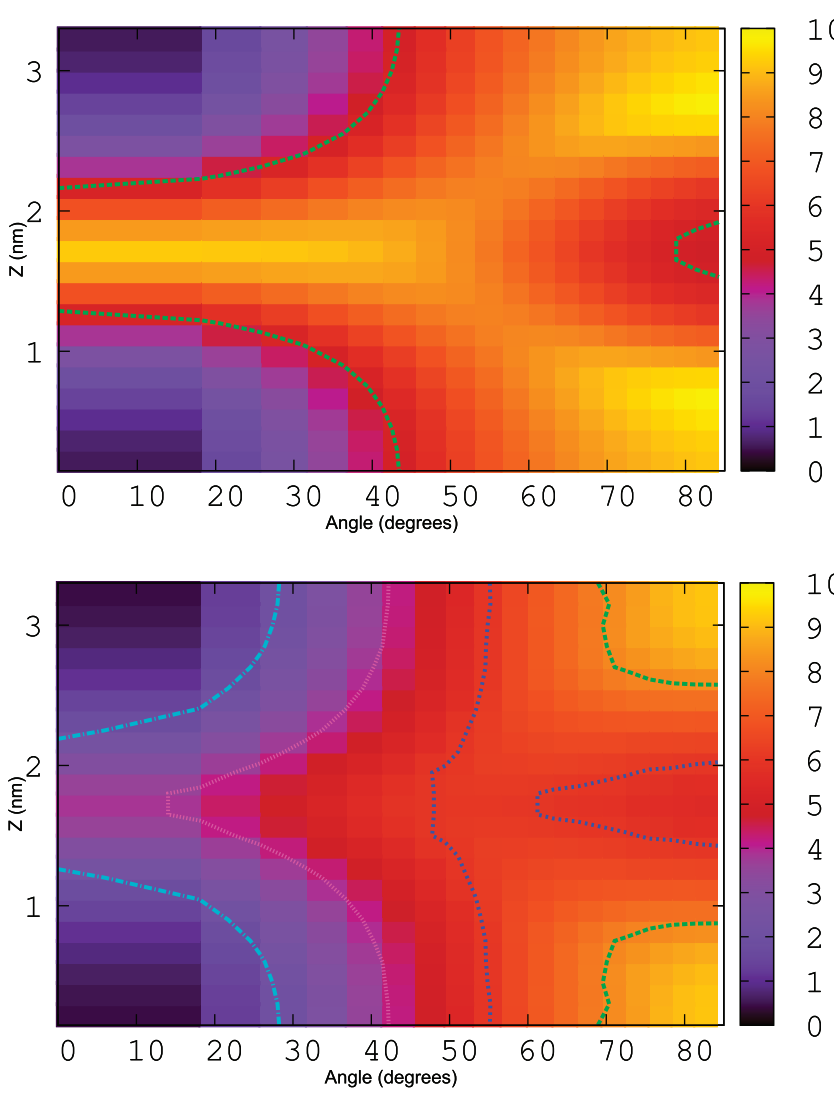

FIG. 15. 2D free-energy surfaces, CG in the upper panel and atomistic in the lower panel. The horizontal axis represents the angle the mesogenic core of the LC molecules make with the $\mathrm{Z}$ direction (also coincides with the director). The vertical axis represents the distance between the centers of molecules and the centers of their nearest Sm-A layers, and the free-energy surface is periodic in this direction. The absolute free-energy minima appear in the lower and upper left corners of the free-energy plot and represent molecules sitting at the center of the Sm-A layer with its axis oriented along the director. A local minimum, though very weak, appears in the CG free-energy surface, at the position of the transition state (midway between the layers and oriented perpendicular to the director) associated with the parking lot translocation. This explains the relative abundance of the parking lot mechanism in the CG simulations compared to the atomistic ones. The translocation mode associated with the lowest free-energy barrier, in the atomistic representation, is a straight jump, while for the CG description, the lowest barrier mode is parking lot. Contour lines are also shown.

supercooled isotropic phase at the state point, where the CG model was parametrized. For this, we compare the standard mean square displacements of the molecules and the reorientational auto-correlation functions. The reorientational autocorrelation function of the molecules is computed using the expression

$$
C_{l}(t)=\left\langle P_{l}\left(\mathbf{u}_{\mathbf{i}}\left(t_{0}\right) \cdot \mathbf{u}_{\mathbf{i}}\left(t_{0}+t\right)\right)\right\rangle_{i, t_{0}},
$$

where $\mathbf{u}_{\mathbf{i}}$ is the unit vector along the mesogenic core of the chosen molecule, "l" denotes the order of the Legendre polynomial, and the angular brackets denote averaging over time-origins and molecules. To compute the speed-up in the translational dynamics, the isotropic MSD for the centers of the individual molecules has been computed. The upper panel of Fig. 16 shows the comparison of the rotational dynamics in the two resolutions, and the lower panel of Fig. 16 shows the comparison of the translational MSD computed in the atomistic and the $\mathrm{CG}$ resolutions. The respective reorientation times have been indicated and the ratio yields the speed-up of $\left(\frac{\tau_{\text {atm }}}{\tau_{C G}}\right.$
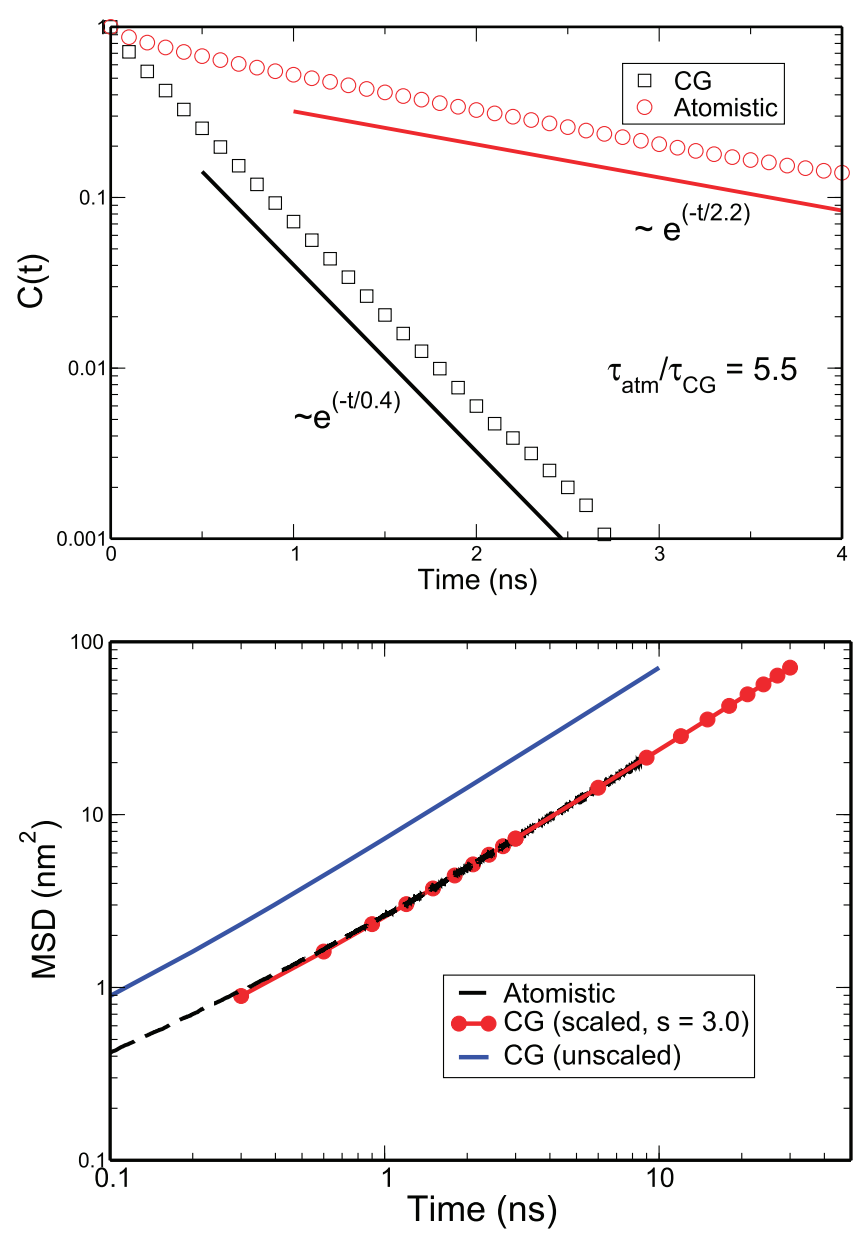

FIG. 16. The comparison of the first-order reorientational correlation functions, shown in the upper panel, calculated from the orientations of the stiff mesogenic core of the LC molecules, simulated in the supercooled, isotropic phase at $460 \mathrm{~K}$, simulated in both atomistic (circles) and CG (squares) representations. A time-scaling factor obtained from the ratio of the correlation times in the two representations connects the intrinsic time scales associated with orientations in the atomistic and CG descriptions. The lower panel shows the unscaled (solid line) CG and the atomistic (dashed line) MSD's calculated from simulations, in both representations, of the supercooled isotropic melt at $460 \mathrm{~K}$. The unscaled CG MSD has been translated along the axis till it coincides with the atomistic MSD. The amount of shift allows us to estimate the time-scaling factor associated with translations in the supercooled melt.

$\sim 5.5$ ), while the translational speed-up is about $\sim 3$. Thus, already in the isotropic state, the reduction of rotational friction is larger than that of translational friction, which has also been observed in non-equilibrium simulations of melts of very short (10 repeat units) polystyrene chains. ${ }^{93}$ The fact that even in the isotropic phase, the translational dynamics is speeded up by a factor of $\sim 3$ again underlines the fact that the reduction in local bead friction here is independent of the environment the molecules find themselves in. A similar speed-up factor has also been observed for the in-plane dynamics of the intraand inter-layer molecules. The effect of larger reduction in rotational friction, compared to translational friction, is also mirrored in the microscopic dynamics in the Sm-A phase, where we have already observed a significant enhancement in the fraction of parking lot translocation (it requires more rotational displacement) in the CG model, compared the atomistic one. 


\section{CONCLUSION}

In this paper, we provide a microscopic understanding of the dynamics and its speed-up for a liquid crystalline azobenzene containing mesogen, simulated in its smectic and isotropic phases, simultaneously in the atomistic and CG resolutions. While the dynamics along the smectic planes is primarily continuous and diffusive, the out-of-plane transport occurs via fast translational hops between two neighbouring smectic layers. These translational hops occur via two different mechanisms in both simulation resolutions. It is important to realise that acceleration in dynamics experienced upon moving from a detailed, atomistic to a CG description results from two separate effects: (a) the acceleration of dynamics due to reduction of bead friction resulting from smoother free-energy landscapes and (b) modification in the relative weights of the microscopic translocation mechanisms induced by intrinsic modifications in certain detailed features of the free-energy landscape, introduced by the act of coarse graining.

Our structure-based CG model, parametrized in the supercooled phase, perfectly reproduces the isotropic to Sm-A phase transition temperature, the order parameter jump, and the density jump of the atomistic system. The out-of-plane transport, as predicted from the CG and the atomistic simulations, can be approximated as a superposition of two (one associated with each mode of translocation) independent continuous time random walks (CTRWs). The CTRW formalism allows us to estimate the amount by which each translocation mode is accelerated when we change from the atomistic to the CG description and we demonstrate that the two independent modes are not accelerated equally. The CTRW formalism also enables us to estimate the relative weights of the individual translocation modes in each representation. Comparing the free-energy surface in the two resolutions, we show that a local minimum corresponding to the transverse interlayer states becomes more prominent in the CG description, thus facilitating a higher percentage of parking lot translocation events. At the same time, a comparison of the speed-up of the short time dynamics shows that the reduction in local bead friction is rather independent of the different environments the molecules find themselves during their temporal evolution.

When one wishes to study the phenomena of structure formation, i.e., after a temperature jump or segregation studies of mixtures of trans and cis 8AB8 molecules, these dynamic inconsistencies, arising from the mismatch of detailed features of the free-energy landscape, might lead to different domain patterns in the course of the simulation for the different models. Not only non-equilibrium properties are affected but also comparisons to experiments on dynamical properties might become difficult. There are a number of recent attempts to improve this situation, and ultimately the goal would be to match the free-energy landscapes between the CG model and the atomistic model (analyzed in CG coordinates), while keeping a close structural equivalence. ${ }^{94,95}$ The latter is needed in order to be able to switch between resolutions as it is highly desirable for many studies. Whether this is possible in general is not clear as this directly relates to basic issues of representability ${ }^{96}$ and transferability upon coarse graining.

\section{SUPPLEMENTARY MATERIAL}

See supplementary material for a discussion on the effect of the coupling time of the thermostat on the dynamics of molecules.

\section{ACKNOWLEDGMENTS}

We thank M. Böckmann, N. Doltsinis, and D. Marx for many stimulating discussions during early stages of this work. Funding from the Volkswagen Foundation and from the SFBTRR146 grant of the German Research Foundation (DFG) for part of this work is gratefully acknowledged. B.M. acknowledges the computational facility availed through TUE-CMS Project No. SR/NM/NS-29/2011(G).

${ }^{1}$ Coarse Graining of Condensed Phase and Biomolecular Systems, edited by G. A. Voth (CRC Press, Boca Raton, FL, 2008).

${ }^{2}$ C. Peter and K. Kremer, Faraday Discuss. 144, 9 (2010).

${ }^{3}$ M. Wilson, editor, "Themed issue on modeling soft matter systems," Soft Matter 5(22), 4341-4584 (30 articles) (2009).

${ }^{4}$ A. F. Voter, Phys. Rev. Lett. 78, 3908 (1997).

${ }^{5}$ A. F. Voter, J. Chem. Phys. 106, 4665 (1997).

${ }^{6}$ A. Laio and M. Parinello, Proc. Natl. Acad. Sci. U. S. A. 99, 12562 (2002).

${ }^{7}$ P. K. Depa and J. K. Maranas, J. Chem. Phys. 123, 094901 (2005).

${ }^{8}$ E. M. Terentjev, Nat. Mater. 1, 149 (2002).

${ }^{9}$ L. Cipelletti, L. Ramos, S. Manley, E. Pitard, D. A. Weitz, E. E. Pashkovsli, and M. Johansson, Faraday Discuss. 123, 237 (2003).

${ }^{10}$ D. Fritz, K. Koschke, V. A. Harmandaris, N. F. A. van der Vegt, and K. Kremer, Phys. Chem. Chem. Phys. 13, 10412 (2011).

${ }^{11}$ B. Hess, S. Leon, N. F. A. van der Vegt, and K. Kremer, Soft Matter 2, 409 (2006).

${ }^{12}$ V. A. Harmandaris, N. P. Adhikari, N. F. A. van der Vegt, and K. Kremer, Macromolecules 39, 6708 (2006).

${ }^{13}$ V. A. Harmandaris, D. Reith, N. F. A. van der Vegt, and K. Kremer, Macromol. Chem. Phys. 208, 2109 (2007).

${ }^{14}$ V. A. Harmandaris, N. P. Adhikari, N. F. A. van der Vegt, K. Kremer, B. A. Mann, R. Voelkl, H. Weiss, and C. C. Liew, Macromolecules 40, 7026 (2007).

${ }^{15}$ V. A. Harmandaris and K. Kremer, Macromolecules 42, 791 (2009).

${ }^{16}$ V. A. Harmandaris and K. Kremer, Soft Matter 5, 3920 (2009).

${ }^{17}$ M. Doi and S. F. Edwards, The Theory of Polymer Dynamics (Oxford University Press, England, 1986).

${ }^{18}$ J. D. Ferry, Viscoelastic Properties of Polymers (John Wiley and Sons, New York, 1980).

${ }^{19}$ Monte Carlo and Molecular Dynamics Simulations in Polymer Science, edited by K. Binder (Oxford University Press, New York, 1995).

${ }^{20}$ D. Fritz, C. R. Herbers, K. Kremer, and N. F. A. van der Vegt, Soft Matter 5, 4556 (2009).

${ }^{21}$ C. Dalgicdir, C. Globisch, M. Sayar, and C. Peter, Eur. Phys. J.: Spec. Top. 225, 1463 (2016).

${ }^{22}$ P. G. de Gennes and J. Prost, The Physics of Liquid Crystals (Clarendon Press, Oxford, 1995).

${ }^{23}$ M. J. Stephen and J. P. Straley, Rev. Mod. Phys. 46, 617 (1974).

${ }^{24}$ W. H. de Jeu, Physical Properties of Liquid Crystalline Materials (Gordon and Breach Science Publishers, 1980).

${ }^{25}$ L. Onsager, Ann. N. Y. Acad. Sci. 51, 627 (1949).

${ }^{26}$ D. Frenkel and B. M. Mulder, Mol. Phys. 55, 1171 (1985).

${ }^{27}$ M. P. Allen, Phys. Rev. Lett. 65, 2881 (1990).

${ }^{28}$ M. R. Wilson, Int. Rev. Phys. Chem. 24(3-4), 421-455 (2005).

${ }^{29}$ D. L. Cheung, S. J. Clark, and M. R. Wilson, Phys. Rev. E 65, 051709 (2002).

${ }^{30}$ D. L. Cheung, S. J. Clark, and M. R. Wilson, J. Chem. Phys. 121, 9131-9139 (2004).

${ }^{31}$ A. J. McDonald and S. Hanna, J. Chem. Phys. 124, 164906 (2006)

${ }^{32}$ I. Cacelli, L. De Gaetani, G. Prampolini, and A. Tani, J. Phys. Chem. B 111, 2130-2137 (2007). 
${ }^{33}$ R. Berardi, L. Muccioli, and C. Zannoni, ChemPhysChem 5, 104-111 (2004).

${ }^{34}$ G. Tiberio, L. Muccioli, R. Berardi, and C. Zannoni, ChemPhysChem 10, 125-136 (2009).

${ }^{35}$ M. F. Palermo, A. Pizzirusso, L. Muccioli, and C. Zannoni, J. Chem. Phys. 138, 204901 (2013).

${ }^{36}$ N. J. Boyd and M. R. Wilson, Phys. Chem. Chem. Phys. 17, 24851-24865 (2015).

${ }^{37}$ B. Mukherjee, L. Delle Site, K. Kremer, and C. Peter, J. Phys. Chem. B 116, 8474-8484 (2012).

${ }^{38}$ L. E. Lombardi, M. A. Marti, and L. Capece, Bioinformatics 32, 1235-1237, (2016).

${ }^{39}$ G. Santangelo, A. Di Matteo, F. Muller-Plathe, and G. Milano, J. Phys. Chem. B 111, 2765-2773 (2007).

${ }^{40}$ F. Thorpe, J. Zhou, and G. A. Voth, J. Phys. Chem. B 112, 13079-13090 (2008).

${ }^{41}$ J. T. Brown, M. P. Allen, E. M. del Rio, and E. de Miguel, Phys. Rev. E 57, 6685-6699 (1998)

${ }^{42}$ E. de Miguel, E. M. del Rio, J. T. Brown, and M. P. Allen, J. Chem. Phys. 105, 4234 (1996).

${ }^{43}$ S. Lee, K. Noda, S. Hirata, and M. Vacha, J. Chem. Phys. Lett. 6, 1403 (2015).

${ }^{44}$ O. D. Lavrentovich, Soft Matter 10, 1264 (2014).

${ }^{45}$ B. Schulz, D. Täuber, J. Schuster, T. Baumgärtel, and C. von Borczyskowski, Soft Matter 7, 7431 (2011).

${ }^{46}$ B. Mukherjee, C. Peter, and K. Kremer, Phys. Rev. E 88, 010502(R) (2013).

${ }^{47}$ A. Patti, D. El Masri, R. van Roij, and M. Djikstra, Phys. Rev. Lett. 103, 248304-1-248304-4 (2009).

${ }^{48}$ M. P. Lettinga and E. Grelet, Phys. Rev. Lett. 99, 197802 (2007).

${ }^{49}$ E. Grelet, M. P. Lettinga, M. Bier, R. van Roij, and P. van der Schoot, J. Phys.: Condens. Matter 20, 494213 (2008).

${ }^{50}$ R. van Roij, P. Bolhuis, B. Mulder, and D. Frenkel, Phys. Rev. E 52, R1277-R1280 (1995).

${ }^{51}$ J. S. van Duijneveldt and M. P. Allen, Mol. Phys. 90, 243-250 (1997)

${ }^{52}$ S. Izvekov and G. A. Voth, J. Chem. Phys. 125, 151101 (2006).

${ }^{53}$ P. Espanol, M. Serrano, and I. Zuniga, Int. J. Mod. Phys. C 8, 899 (1997).

${ }^{54}$ H. Qian, C. C. Liew, and F. Muller-Plathe, Phys. Chem. Chem. Phys. 11, 1962 (2009).

${ }^{55}$ C. Hijon, P. Espanol, E. Vanden-Eijnden, and R. Delgado-Buscalioni, Faraday Discuss. 144, 301 (2010).

${ }^{56}$ P. Espanol and I. Zuniga, Phys. Chem. Chem. Phys. 13, 10538 (2011).

${ }^{57}$ C. Fu, P. M. Kulkarni, M. S. Shell, and L. G. Leal, J. Chem. Phys. 139, 094107 (2013).

${ }^{58}$ S. Trement, B. Schnell, L. Petitjean, M. Couty, and B. Rousseau, J. Chem. Phys. 140, 134113 (2014).

${ }^{59}$ Z. Li, X. Bian, B. Caswell, and G. E. Karniadakis, Soft Matter 10, 8659 (2014).

${ }^{60}$ S. Markutsya and M. H. Lamm, J. Chem. Phys. 141, 174107 (2014).

${ }^{61}$ Z. Li, H. S. Lee, E. Darve, and G. E. Karniadakis, J. Chem. Phys. 146, 014104 (2017).

${ }^{62}$ A. Davtyan, J. F. Dama, G. A. Voth, and H. C. Andersen, J. Chem. Phys. 142, 154104 (2015).
${ }^{63}$ A. Davtyan, G. A. Voth, and H. C. Andersen, J. Chem. Phys. 145, 224107 (2016).

${ }^{64}$ S. Izvekov, J. Chem. Phys. 146, 124109 (2017).

${ }^{65}$ R. Zwanzig, J. Stat. Phys. 9, 215 (1973).

${ }^{66}$ R. Zwanzig, Nonequilibrium Statistical Mechanics (Oxford University Press, Oxford, 2001).

${ }^{67}$ S. Izvekov and G. A. Voth, J. Chem. Phys. 123, 134105 (2005).

${ }^{68}$ S. Izvekov and G. A. Voth, J. Phys. Chem. B 109, 2469 (2005).

${ }^{69}$ W. G. Noid, J. W. Chu, G. S. Ayton, and G. A. Voth, J. Phys. Chem. B 111, 4116 (2007).

${ }^{70}$ W. G. Noid, J. W. Chu, G. S. Ayton, V. Krishna, S. Izvekov, G. A. Voth, A. Das, and H. C. Andersen, J. Chem. Phys. 128, 244114 (2008).

${ }^{71}$ W. G. Noid, P. Liu, Y. Wang, J. Chu, G. S. Ayton, S. Izvekov, H. C. Andersen, and G. A. Voth, J. Chem. Phys. 128, 244115 (2008).

${ }^{72}$ W. Tschoep, K. Kremer, O. Hahn, J. Batoulis, and T. Bürger, Acta Polym. 49, 75 (1998).

${ }^{73}$ R. Kutner and J. Masoliver, Eur. Phys. J. B 90, 50 (2017).

${ }^{74}$ E. W. Montroll and G. H. Weiss, J. Math. Phys. 6, 167 (1965).

${ }^{75}$ L. Berthier, D. Chandler, and J. P. Garrahan, Eur. Phys. Lett. 69, 320 (2005).

${ }^{76}$ P. Chaudhuri, L. Berthier, and W. Kob, Phys. Rev. Lett. 99, 060604 (2007).

${ }^{77}$ P. Chaudhuri, Y. Gao, L. Berthier, M. Kilfoil, and W. Kob, J. Phys.: Condens. Matter 20, 244126 (2008).

${ }^{78}$ D. Chandler and J. P. Garrahan, Annu. Rev. Phys. Chem. 61, 191 (2010).

${ }^{79}$ M. Warren and J. Rottler, Eur. Phys. Lett. 88, 58005 (2009).

${ }^{80} \mathrm{~J}$. Helfferich, F. Ziebert, S. Frey, H. Meyer, J. Farago, A. Blumen, and J. Baschnagel, Phys. Rev. E 89, 042603 (2014).

${ }^{81}$ J. Helfferich, K. Vollmayr-Lee, F. Ziebert, H. Meyer, and J. Baschnagel, Eur. Phys. Lett. 109, 36004 (2015).

${ }^{82}$ R. Pastore, A. Coniglio, and M. P. Ciamarra, Soft Matter 10, 5724 (2014).

${ }^{83}$ R. Pastore, A. Coniglio, and M. P. Ciamarra, Sci. Rep. 5, 11770 (2015).

${ }^{84}$ M. P. Ciamarra, R. Pastore, and A. Coniglio, Soft Matter 12, 358 (2016).

${ }^{85}$ B. Hess, C. Kutzner, D. van der Spoel, and E. Lindahl, J. Chem. Theory Comput. 4, 435 (2008).

${ }^{86}$ M. Boeckmann, C. Peter, L. Delle Site, N. L. Doltsinis, K. Kremer, and D. Marx, J. Chem. Theory Comput. 3, 1789 (2007).

${ }^{87}$ G. Bussi, D. Donadio, and M. Parinello, J. Chem. Phys. 126, 014101 (2007).

${ }^{88}$ Stochastic Processes, edited by S. M. Ross (John Wiley and Sons, 1995).

${ }^{89}$ An Introduction to Probability Theory and Its Applications, edited by W. Feller (John Wiley and Sons, 2008).

${ }^{90}$ Theory of Simple Liquids, edited by J. P. Hansen and I. R. McDonald (Academic Press, 1986).

${ }^{91}$ B. J. Berne and G. D. Harp, "On the Calculation of Time Correlation Functions, " in Advances in Chemical Physics, Volume 17, edited by I. Prigogine and S. A. Rice (John Wiley \& Sons, Inc., Hoboken, NJ, 1970).

${ }^{92}$ L. van Hove, Phys. Rev. 95, 249 (1954).

${ }^{93}$ C. Baig and V. A. Harmandaris, Macromolecules 43, 3156 (2010).

${ }^{94}$ J. Rudzinski and T. Bereau, Eur. Phys. J.: Spec. Top. 225, 1373 (2016).

${ }^{95}$ J. Rudzinski, T. Bereau, and K. Kremer, J. Chem. Phys. 144, 051102 (2016).

${ }^{96}$ J. W. Wagner, J. F. Dama, A. Durumeric, and G. A. Voth, J. Chem. Phys. 145, 044108 (2016). 